\title{
A Pragmatist View of the Metaphysics of Entanglement
}

\author{
Richard Healey \\ University of Arizona
}

May 13, 2015

\begin{abstract}
Quantum entanglement is widely believed to be a feature of physical reality with undoubted (though debated) metaphysical implications. But Schrödinger introduced entanglement as a theoretical relation between representatives of the quantum states of two systems. Entanglement represents a physical relation only if quantum states are elements of physical reality. So arguments for metaphysical holism or nonseparability from entanglement rest on a questionable view of quantum theory. Assignment of entangled quantum states predicts experimentally confirmed violation of Bell inequalities. Can one use these experimental results to argue directly for metaphysical conclusions? No. Quantum theory itself gives us our best explanation of violations of Bell inequalities, with no superluminal causal influences and no metaphysical holism or nonseparability - but only if quantum states are understood as objective and relational, though prescriptive rather than ontic. Correct quantum state assignments are backed by true physical magnitude claims: but backing is not grounding. Quantum theory supports no general metaphysical holism or nonseparability; though a claim about a compound physical system may be significant and true while similar claims about its components are neither. Entanglement may well have have few, if any, first-order metaphysical implications. But the quantum theory of entanglement has much to teach the metaphysician about the roles of chance, causation, modality and explanation in the epistemic and practical concerns of a physically situated agent.
\end{abstract}

Acknowledgement 1 This paper benefited from discussions withJenann Ismael and David Glick and helpful suggestions from two reviewers. I thank the Yetadel Foundation for support during the time it was written. 


\section{Introduction}

Many philosophers believe that quantum entanglement has important metaphysical implications. In this paper I explain why I disagree. My disagreement rests on two claims. First, entanglement is not a physical relation between physical systems but a feature of the way certain quantum states must be represented. Second, even though they are objective, these and other quantum states are not novel physical objects, fields or magnitudes: nor do they represent physical relations or properties of objects to which they are assigned. It's quicker to say systems are entangled than that their quantum states must be mathematically represented in a certain way, but that is almost always what physicists mean by entanglement. That is what Schrödinger meant when he introduced the concept, as I explain in section 2. Section 3 is a brief introduction to different conceptions of entanglement, so conceived.

It was Einstein who first perceived metaphysical intimations of entanglement in what he took to be the "orthodox" view of quantum states. Section 4 explains where he discerned them, and why Einstein rejected this "orthodox" view because of what he took to be its metaphysical implications. I also state (but do not endorse) some metaphysical principles of holism and nonseparability I have explained elsewhere. Section 5 shows how, by taking quantum state entanglement to imply a physical relation between physical systems, the view Einstein called "orthodox" can be seen to imply metaphysical holism and nonseparability. Section 6 considers whether other "unorthodox" views of the quantum state have similar implications. Einstein suggested one view that does not, but Bell and others have shown what's wrong with this view.

Schrödinger entertained the view that a quantum state represents not a physical system but our knowledge of it - a view that has at least as strong a claim to be called orthodox. When purged of subjectivism while acknowledging the importance of application, this becomes the view that a quantum state is objective but relative to the physical situation of any hypothetical agent in a position to assign it. In section 7 I sketch such a view I have advanced elsewhere, emphasizing the role of a quantum state as authoritative source of reliable advice on the significance and credibility of certain claims about physical systems. Section 8 shows how by adopting this view one can use the assignment of an entangled state to explain patterns of correlation in violation of Bell inequalities with no instantaneous action at a distance.

In section 9 I explain why in this view entangled quantum states require no grounding, and why there is nothing nonseparable that requires or admits explanation in terms of a metaphysical grounding relation. Section 10 shows what one can learn about chance, causation and explanation from the application of quantum theory to explain violations of Bell inequalities. I conclude by pointing out that the advantages of understanding chance, causation, explanation and quantum states in terms of the functions of these concepts in thinking about and acting in the physical world support a more general pragmatism. This seeks philosophical illumination by asking not what in the world corresponds to these concepts, but what in the world would make these concepts useful in the 
epistemic and practical activities of physically situated, and so informationally deprived, agents like us. In this light metaphysics is nowhere to be seen.

\section{How it all Began}

As is well known, Schrödinger introduced the concept he named entanglement in the following passage:

When two systems, of which we know the states by their respective representatives, enter into temporary physical interaction due to known forces between them, and when after a time of mutual influence the systems separate again, then they can no longer be described in the same way as before, viz. by endowing each of them with a representative of its own. I would not call that one but rather the characteristic trait of quantum mechanics, the one that enforces its entire departure from classical lines of thought. By the interaction the two representatives (or $\psi$-functions) have become entangled. ( [55], p. 555)

Writing in German later the same year he used the word 'Verschränkung'. Unlike the English word 'entanglement', this German word is used to express clasping of hands, crossing of legs and folding of arms - in each case an action intimately connecting just two objects. ${ }^{1}$ Schrödinger [56] first applied the word 'Verschränkung' to predictions and to knowledge, then to variables like position and momentum, to measurements, and to the "expectation catalogs" provided by quantum state assignments. In none of the five parts of his two major 1935-6 publications on the interpretation of quantum mechanics did he apply either 'entangled' or 'verschränkt' directly to physical systems.

It is somewhat less well known that Schrödinger's interest in entanglement was sparked by the famous EPR [16] paper and further enflamed by a fascinating correspondence with Einstein following its publication (see Fine [18], chapters 3,5). Schrödinger first used the word 'Verschränkung' in print on page 827 of the paper in which he presented his famous "cat paradox" [56]. ${ }^{2} \mathrm{He}$ acknowledges the influence of the EPR paper in the following footnote.

"The appearance of this work motivated the present - shall I say lecture or general confession?" [op. cit., p. 845]

\footnotetext{
${ }^{1}$ By this word choice he unconsciously anticipated the monogamy of (maximal) entanglement.

${ }^{2}$ Interestingly he also used the word 'verheddert' on the same page to describe the way a system's $\psi$-function gets tangled up with that of an apparatus during a measurement. He uses only 'Verschränkung' and 'verschränkt' throughout the rest of the paper.
} 


\section{What is Entanglement?}

For Schrödinger, entanglement presented a challenge to our understanding of quantum theory. ${ }^{3}$ Today physicists regard it as a resource to be exploited in technological applications of quantum information and communication. But disputes continue on how to understand quantum theory, and (to echo the title of an important recent article) there still remain some puzzles and unresolved issues about quantum entanglement itself (see Earman [12]).

Schrödinger applied the concept only to pure quantum states of two previously interacting systems, or rather to their representations in non-relativistic quantum mechanics by $\psi$-functions in a Hilbert space of vector states $|\psi\rangle \cdot{ }^{4} \mathrm{In}$ that context one can express the condition as follows

$$
\Psi_{a b} \neq \psi_{a} \psi_{b} \quad \text { (Vector Entanglement) }
$$

where $\Psi_{a b}$ represents a pure quantum state assigned to the composite system $a b,\left\{\psi_{a}, \psi_{b}\right\}$ is any ordered pair of $\psi$-functions from the Hilbert spaces of vectors used to represent states assigned to systems $a, b$ respectively, and the multiplication of $\psi$-functions on the right corresponds to their tensor product $\otimes$ when considered as vectors in these spaces. Each of $a, b$ is here assumed to be a massive object - either a particle or itself composed of particles - so the system $a b$ is a massive composite object.

A hydrogen atom can serve as an example. If one takes $a$ to be its electron and $b$ its nuclear proton and treats the atom non-relativistically (ignoring spin), then neither electron nor proton can be assigned a pure quantum state even while the atom is assumed to be isolated and so assigned its lowest energy state. This may seem surprising if one assumes that the $\psi$-function

$$
\psi(\mathbf{r})=\frac{1}{\sqrt{\pi}}\left(\frac{1}{a_{0}}\right)^{\frac{3}{2}} \exp \left(-r / a_{0}\right)
$$

represents the quantum state of the electron (where $a_{0}=\hbar^{2} / m e^{2}$ is the radius of the first "Bohr orbit"). But $\mathbf{r}$ here does not represent the electron's position $\mathbf{r}_{e}$ but its position relative to the nucleus $\left(\mathbf{r}=\mathbf{r}_{e}-\mathbf{r}_{p}\right)$, and $m$ represents the

${ }^{3}$ Or even to its adequacy:

Indubitably the situation described here is, in present quantum mechanics,

a necessary and indispensable feature. The question arises, whether it is so in

Nature too. I am not satisfied about there being sufficient experimental evidence

for that. ([57], p. 451)

Entanglement is still a necessary and indubitable feature of quantum theory - a feature that has by now received exhaustive experimental confirmation.

${ }^{4}$ The concept is now regularly applied to states of two or more systems that have never interacted, and even retrospectively to systems that no longer exist (e.g. in entanglementswapping scenarios, some of which include delayed choice). It has been generalized to apply to other kinds of representations of states, also in relativistic quantum mechanics and quantum field theory. 
so-called reduced mass of the electron $m=\frac{m_{e} m_{p}}{m_{e}+m_{p}}$. The atomic wave function is not entangled when expressed as the product

$$
\Psi(\mathbf{R}, \mathbf{r})=\psi(\mathbf{r}) \varphi(\mathbf{R})
$$

in which $\varphi(\mathbf{R})$ is a pure state assigned to the atom's center of mass

$$
\mathbf{R}=\frac{m_{p} \mathbf{r}_{p}+m_{e} \mathbf{r}_{e}}{m_{p}+m_{e}}
$$

This example illustrates several important points about entanglement. The first is that (Vector Entanglement) expresses entanglement as a relation between representatives of quantum states, not between systems. The second is that in order to use a mathematical relation like (Vector Entanglement) to infer a relation between subsystems one must first associate each representative with a system by specifying of what mathematical structure that representative is an element: The example shows that just saying what structure the composite system's representative "lives in" may fail uniquely to determine its subsystems or how their states might be represented. ${ }^{5}$

But isn't it obvious that the real subsystems of a hydrogen atom are its electron and proton, not the center-of-mass subsystem and the reduced-masselectron subsystem? This raises the third important point about entanglement. In order to promote a mathematical relation like (Vector Entanglement) to a physical (or metaphysical) relation between physical systems, one must appeal to some independent criterion of what makes a system physical. If one restricts attention to the domain of non-relativistic quantum mechanics, that may seem not so hard to come by: A system is physical only if it is composed of particles - atoms, electrons, protons and such. But contemporary generalizations of Schrödinger's concept are now regularly applied outside of that domain - to massless photons, to other quanta of relativistic quantum field theories of the Standard Model, to open bounded regions of spacetime in so-called local quantum physics, and more abstractly in the algebraic approach to quantum physics where ontological questions are often dismissed or swept under the rug.

If $a, b$ are indistinguishable fermions, such as any two electrons, then (Vector Entanglement) implies that their representatives are always entangled, since their joint representative must change sign under exchange of the labels ' $a$ ', $b^{\prime}$ ': $\left|\Psi_{a b}\right\rangle=-\left|\Psi_{b a}\right\rangle$. The requirement that the representative of any such set of indistinguishable fermions be totally antisymmetric (i.e. change sign under any single swap of labels) has been used to argue that all indistinguishable fermions of the same type are always entangled, whether or not they have interacted.

...there is a universal correlation of the EPR type which we do not have to cleverly set up, it is simply the total antisymmetrization

\footnotetext{
${ }^{5}$ As the example shows, a Hilbert space generally has multiple inequivalent decompositions as a tensor product of Hilbert spaces.
} 
of a many fermion state, which does correlate the electrons of my body with those of any inhabitant of the Andromeda galaxy. (LevyLeblond [43])

But this is best seen as challenging the adequacy of (Vector Entanglement) as a criterion for a pair of indistinguishable fermions to be entangled.

A more important reason to formulate a better criterion is provided by Gisin's theorem [24]. This is usually stated as follows: All non-product (pure) states of two systems violate a Bell inequality (specifically, a CHSH [9] inequality.) At first sight this theorem provides a strong reason supporting adoption of (Vector Entanglement) as necessary and sufficient for entanglement of two pure vector state representatives, because violation of a Bell inequality is a clear sign of non-classical behavior. But suppose one were to prepare two unexcited atoms of lithium-6, one in a laboratory in Leiden with $z$-spin up, the other in a laboratory in Rio with $z$-spin down, so their translational states are effectively representable by non-overlapping $\psi$-functions or orthogonal state vectors $|L\rangle,|R\rangle$. Lithium-6 is an uncommon isotope of this element. An unexcited atom of Li-6 has spin 1. Because the atoms are indistinguishable fermions, their total state must be antisymmetrized as follows

$$
\frac{1}{\sqrt{2}}\left(|L\rangle_{a}|\uparrow\rangle_{a}|R\rangle_{b}|\downarrow\rangle_{b}-|R\rangle_{a}|\downarrow\rangle_{a}|L\rangle_{b}|\uparrow\rangle_{b}\right) .
$$

This is not a product state, so Gisin's theorem apparently implies that it must violate a CHSH inequality. In fact it does not, since the Indistinguishability Postulate ensures that the only observables on the total system are those that commute with operators inducing permutation of state spaces (here corresponding to the labels $a, b)$ : a measurement of $z$-spin-component in Leiden is not representable by an operator in either the $a$ or the $b$ space but by the operator $\hat{S}_{a z} \otimes \hat{1}_{b}+\hat{1}_{a} \otimes \hat{S}_{b z}$ on the total $a b$ space. To "prove" Gisin's theorem for indistinguishable particles one would need to appeal to the existence of observables ruled out by the Indistinguishability Postulate.

Several authors ${ }^{6}$ have proposed a revised criterion for Indistinguishable Fermion Entanglement (IFE) that may be expressed as follows:

The states of systems of indistinguishable fermions $S_{a}$ and $S_{b}$ composing system $S_{a b}=S_{a}+S_{b}$ assigned state $\left|\Psi_{a b}\right\rangle$ are entangled if and only if $\left|\Psi_{a b}\right\rangle$ canot be obtained by antisymmetrizing a product $\left|\psi_{a}\right\rangle \otimes\left|\psi_{b}\right\rangle$.

A simple revision of Gisin's proof now shows that all states of two identical fermions that satisfy this criterion of entanglement violate a $\mathrm{CHSH}$ inequality. Here is one such state that may be assigned to a pair of unexcited atoms of lithium-6 with opposite $z$-spins, one in a laboratory in Leiden, the other in a laboratory in Rio

$$
\frac{1}{\sqrt{2}}\left(|\uparrow\rangle_{a}|\downarrow\rangle_{b}+|\downarrow\rangle_{a}|\uparrow\rangle_{b}\right) \otimes\left(|L\rangle_{a}|R\rangle_{b}-|R\rangle_{a}|L\rangle_{b}\right) .
$$

\footnotetext{
${ }^{6}[22],[21],[42],[8]$.
} 
With very high probability, statistics collected in suitable experiments on lithium atoms assigned state (5) would violate a CHSH inequality, while statistics collected in an experiment on atoms assigned state (4) would never violate that inequality.

Philosophers have often used the following vector representing the spin state of two spin- $\frac{1}{2}$ particles to illustrate entanglement

$$
\frac{1}{\sqrt{2}}\left(|\uparrow\rangle_{a}|\downarrow\rangle_{b}-|\downarrow\rangle_{a}|\uparrow\rangle_{b}\right)
$$

This EPR-Bohm state may be assigned to a pair of hydrogen atoms formed by dissociation of a hydrogen molecule. In this situation each atom acts as a boson but (6) is antisymmetrized, despite the fact that the state assigned to a system of indistinguishable bosons must be symmetrized - it must remain the same under exchange of labels like $a, b$. But (6) does not represent the total state of the pair of hydrogen atoms. Assigning the following symmetrized state respects the bosonic character of the hydrogen atoms in a pair, one in Leiden, the other in Rio

$$
\frac{1}{\sqrt{2}}\left(|\uparrow\rangle_{a}|\downarrow\rangle_{b}-|\downarrow\rangle_{a}|\uparrow\rangle_{b}\right) \otimes\left(|L\rangle_{a}|R\rangle_{b}-|R\rangle_{a}|L\rangle_{b}\right) .
$$

Much more could be said about how to define entanglement of states of two or more systems in various forms of quantum theory that use different mathematical objects to represent quantum states of different types of system (see [12]). But it is time to ask what kind of gold metaphysicians have sought to extract from the mathematical ore of quantum entanglement.

\section{Metaphysical Prospects}

It was not a philosopher but a physicist who first perceived the metaphysical intimations of quantum entanglement - but rejected them! Bohr and Schrödinger each saw measurement as uniting object and apparatus into a kind of indivisible whole, though in different ways. Einstein introduced a principle of separation and argued that it was incompatible with the orthodox view of quantum theory.

But the holism that Schrödinger located in quantum entanglement was not metaphysical but epistemological. Writing in English, this is how he first expressed it in 1935:

...the best possible knowledge of a whole does not necessarily include the best possible knowledge of all its parts, even though they may be entirely separated and therefore virtually capable of being "best possibly known", i.e. of possessing, each of them, a representative of its own. ([55], p. 555)

He further elaborates in his "cat paper" 
Let us pause for a moment. This result in its abstractness actually says it all: Best possible knowledge of a whole does not necessarily include the same for its parts. .... The whole is in a definite state, the parts taken individually are not.

"How so? Surely a system must be in some sort of state." "No. State is $\psi$-function, is maximal sum of knowledge. I didn't necessarily provide myself with this, I may have been lazy. Then the system is in no state."

"Fine, but then too the agnostic prohibition of questions is not yet in force and in our case I can tell myself: the subsystem is already in some state, I just don't know which."

"Wait. Unfortunately no. There is no 'I just don't know.' For as to the total system, maximal knowledge is at hand..."

The insufficiency of the $\psi$-function as model replacement rests solely on the fact that one doesn't always have it. If one does have it, then by all means let it serve as description of the state. But sometimes one does not have it, in cases where one might reasonably expect to. And in that case, one dare not postulate that it "is actually a particular one, one just doesn't know it"; the above-chosen standpoint forbids this. "It" is namely a sum of knowledge; and knowledge, that no one knows, is none.

([56], p. 827: this English translation by J.D. Trimmer, reprinted in $[61]$, p. 161. Italics in original.)

By 'the above chosen standpoint' Schrödinger here refers to the view that a representative ( $\psi$-function) of a quantum state represents a catalog of expectations concerning possible future measurement outcomes. The italicized sentence explicitly rejects the view that a $\psi$-function models an element of physical reality in the way that a function on $3 n$-dimensional configuration space (or $6 n$ dimensional phase space) could be taken to model the behavior of a system of $n$ particles in classical mechanics.

Bohr also rejected this view of the $\psi$-function. In his later writings he often used the term 'wholeness' when discussing quantum measurement.

... the essential wholeness of a proper quantum phenomenon finds indeed logical expression in the circumstance that any attempt at its well-defined subdivision would require a change in the experimental arrangement incompatible with the appearance of the phenomenon itself. ([7], p. 72)

Earlier he had applied the term 'individuality' in a similar way, but more widely to apply also to atomic processes other than measurement. For example, he says that the essence of quantum theory

...may be expressed in the so-called quantum postulate, which attributes to any atomic process an essential discontinuity, or rather individuality, completely foreign to the classical theories and symbolized by Planck's quantum of action. ([5], p. 53) 
As far as I know Bohr neither uses 'entangle' or its derivatives nor considers analyses like Schrödinger's that apply quantum theory to a measurement interaction. Indeed his remarks about observation strongly suggest that he would consider any such application mistaken. Instead his writings repeatedly trace quantum "wholeness" back to "the quantum postulate" introduced by Planck prior to the development of matrix and wave mechanics. As far as I can tell, Bohr did not himself seek to draw metaphysical conclusions from the quantum theory of entanglement. But Einstein seems to have thought otherwise.

Of the "orthodox" quantum theoreticians whose position I know, Niels Bohr's seems to me to come nearest to doing justice to the problem. Translated into my own way of putting it, he argues as follows: If the partial systems $A$ and $B$ form a total system which is described by its $\psi$-function $\psi_{A B}$, there is no reason why any mutually independent existence (state of reality) should be ascribed to the partial systems $A$ and $B$ viewed separately, not even if the partial systems are spatially separated from each other at the particular time under consideration. The assertion that, in this latter case, the real situation of $B$ could not be (directly) influenced by any measurement taken on $A$ is, therefore, within the framework of quantum theory, unfounded and (as the paradox shows) unacceptable. ([15], pp. 681-682)

This is how Einstein seems to have understood the following crucial passage in Bohr's reply to the EPR paper, in which he takes Bohr's system to comprise the total system $A B$.

Of course there is in a case like that just considered no question of a mechanical disturbance of the system under investigation during the last critical stage of the measuring procedure. But even at this stage there is essentially the question of an influence on the very conditions which define the possible types of predictions regarding the future behavior of the system. ([6], p. 700)

But notice that Bohr speaks not about states of reality but only of "possible predictions". Here and elsewhere he is concerned more about the conditions presupposed by meaningful description of physical systems than about the nature of any reality to which such statements might correspond. If that's right, Einstein here turns Bohr into metaphysician against his wishes.

By contrast, Einstein acknowledges his own metaphysical commitments and explains how they influence his attitude toward quantum theory.

What does not satisfy me in [quantum] theory, from the standpoint of principle, is its attitude towards that which appears to me to be the programmatic aim of all physics: the complete description of any (individual) real situation (as it supposedly exists irrespective 
of any act of observation or substantiation). Whenever the positivistically inclined modern physicist hears such a formulation his reaction is that of a pitying smile. He says to himself: "there we have the naked formulation of a metaphysical prejudice, empty of content, a prejudice, moreover, the conquest of which constitutes the major epistemological achievement of physicists within the last quarter-century. Has any man ever perceived a 'real physical situation'? How is it possible that a reasonable person could today still believe that he can refute our essential knowledge and understanding by drawing up such a bloodless ghost?" Patience! The above laconic characterisation was not meant to convince anyone; it was merely to indicate [a] point of view. (op.cit. p. 667)

Einstein took as a key tenet of the "orthodox" quantum theoreticians (among whom he included Bohr) that the description of a system by means of the $\psi$-function is complete. The EPR argument, as well as the modified argument that Einstein himself repeated after expressing dissatisfaction with the way the EPR paper turned out ${ }^{7}$, was intended to show that this tenet is false. Einstein's modified argument first emerged in a letter he wrote to Schrödinger dated June 19, 1935. This argument assumed a principle governing the states of systems that have interacted only in the past which Howard ([36], p. 180) translates as follows:

Real State Separability Principle: The real state of the pair $A B$ consists precisely of the real state of $A$ and the real state of $B$, which states have nothing to do with one another.

By accepting this principle Einstein rejected a kind of holism. He argued that acceptance of such holism is the only way to hold on to the "orthodox" tenet that the description of a system by means of the $\psi$-function is complete. That is why he thought orthodox quantum theorists were committed to a kind of metaphysical holism. But since Bohr denied any descriptive role to the $\psi$-function Einstein was wrong to include him in the company of the "orthodox" theoreticians. ${ }^{8}$

Whether or not a $\psi$-function completely describes a system to which it is assigned, one can formulate a more general principle applicable to state assignments in any physical theory.

State Separability: The state assigned to a compound physical system at any time is supervenient on the states then assigned to its component subsystems.

There are assignments of quantum states to compound systems that violate this principle, including (5) and (7). Whether this has any metaphysical implications depends on the nature of quantum states.

\footnotetext{
${ }^{7}$ See $([18]$, chapter 3$)$.

${ }^{8}$ Einstein was right to consider Dirac, von Neumann and others following them "orthodox" in his sense because they accepted the descriptive completeness of the theory.
} 
In common parlance as well as classical mechanics the term 'state' is generally used to connote the condition of something, either at one moment or as this varies over a period of time: a system's state says how it is - what are the properties and relations of the things that make it up. In this usage, to specify the state of a system is to describe it. If this is the function of state assignment, then State Separability is incompatible with holism, understood as follows:

Physical Property Holism: There is some set of physical objects from a domain $D$ subject only to type $P$ processes, not all of whose qualitative intrinsic physical properties and relations supervene on qualitative intrinsic physical properties and relations in the supervenience basis of their basic physical parts (relative to $D$ and $P$ ).

It is perhaps not too much of a stretch to read Einstein as rejecting such metaphysical holism, while taking the "orthodox" quantum theoreticians to be committed to it.

Elsewhere Einstein ([14], pp. 323-4) stated a closely related principle that appealed to spatiotemporal location as a condition of separation.

An essential aspect of [the] arrangement of things in physics is that they lay claim, at a certain time, to an existence independent of one another, provided these objects 'are situated in different parts of space'. (Transl. Howard [37])

Here are two metaphysical principles suggested by this (cf. my [29]).

Spatial Separability: The qualitative intrinsic physical properties of a compound system are supervenient on those of its spatially separated component systems together with the spatial relations among these component systems.

Spatiotemporal Separability: Any physical process occupying spacetime region $R$ supervenes upon an assignment of qualitative intrinsic physical properties at spacetime points in $R$.

To deny either of these two principles is to accept a corresponding variety of metaphysical nonseparability.

Of all the "founding fathers" it was only Einstein who perceived metaphysical intimations of entanglement concerning holism and nonseparability. But he denied that entanglement implied any kind of holism and nonseparability and used this denial as a premise in his arguments against a view of quantum theory he took to have such metaphysical implications. This may not have been Bohr's view, but Einstein was not wrong to call it "orthodox". Dirac and von Neumann maintained the descriptive completeness of quantum description and many others have followed them. This now includes a number of philosophers who have drawn the kind of metaphysical conclusions that Einstein rejected. 


\section{5 "Orthodox" Prospectors}

Quantum entanglement is in the first instance a mathematical relation between mathematical objects that may be used to represent the states of systems. To promote this to a physical relation such mathematical objects must be taken to represent something physical, in which case these are physical states describing physical systems. On the view I shall follow Einstein in calling "orthodox", to assign a quantum state to a system is to describe it, indeed to describe it completely.

This is a qualified sense of 'completeness'. Assigning state (2) to a hydrogen atom does not describe the quark constituents of its nuclear proton, and while (2) includes the magnitudes $m_{p}, m_{e}, e$ it does not say that these are (in order) the mass of the proton, and the mass and charge of the electron. To assign this state is to treat a hydrogen atom as a non-relativistic system of two structureless, spinless particles. This idealization is clearly incomplete because it omits features of real hydrogen atoms.

The "orthodox" view is that this description is nevertheless complete in the sense that it cannot be completed by specifying the values of all classical dynamical variables pertaining to an idealized system of this type, or of any other "hidden" dynamical variables. One way to make this more precise is to adopt a semantic rule Fine [19] calls the eigenstate-eigenvalue link: this holds that a dynamical variable of a system has an eigenvalue if and only if the state of the system is an eigenstate of the corresponding operator with that eigenvalue (or a mixture of such eigenstates). Dirac [11] and von Neumann [59] arguably both adopted this rule in their seminal texts, and something like it continues to be implicit (though unremarked) in many contemporary presentations of quantum theory.

Applied to state (2) the eigenstate-eigenvalue link implies that the hydrogen atom has a precise internal energy $(-13.6$ electron volts) and (ignoring mathematical niceties) a precise center of mass momentum $\mathbf{P}$ if $\varphi(\mathbf{R}) \propto$ $\exp (i \mathbf{P} . \mathbf{R} / \hbar)$, but that it has no definite center of mass position; and its electron has no definite location, speed, kinetic energy or potential energy relative to its proton. Applied to (5) (or (7)) the eigenstate-eigenvalue link has the surprising implications that neither atom has a definite spin component in any direction even though their total spin (as well as its component in any direction) is zero; and that neither atom is anywhere in particular - neither in Leiden nor in Rio. By contrast, if the eigenstate-eigenvalue link is applied to (4) it has the expected implications that a lithium atom in Leiden has spin up with respect to the $z$-axis while a lithium atom in Rio has spin down.

Suppose one takes the eigenstate-eigenvalue link to specify the real state of a system. Then the failure of State Separability implies that the Real State Separability Principle is false, arguably demonstrating Physical Property Holism: In the state (7) the hydrogen atoms are the basic physical parts of the pair they compose, whose real state includes a property having zero spin that

does not supervene on qualitative intrinsic physical properties and relations of those atoms (including their spins and spatial relations). 
The "orthodox" argument for nonseparability (i.e. the denial of Spatial Separability and/or Spatiotemporal Separability) is not quite so straightforward. To use the supposed falsity of the Real State Separability Principle to refute Spatial Separability one needs an example of spatially separated subsystems. But in the "orthodox" view, the atoms in a state like (5) or (7) have no definite spatial location! Nevertheless, if the spatial $\psi$-functions of the states $|L\rangle_{a},|R\rangle_{b}$ are sufficiently localized ${ }^{9}$, (something like) the eigenstate-eigenvalue link will imply that they are separated by a (fairly) determinate distance. The atoms may then be claimed to be spatially separated even though the qualitative intrinsic physical properties (e.g. the spin) of the pair are not supervenient on those of each individual atom, even together with their spatial relations.

\section{Unorthodox Interpretations}

Einstein's own argument that the quantum description is incomplete did not depend on the eigenstate-eigenvalue link. It targeted a version of "orthodoxy" according to which quantum reality is completely and directly described by the $\psi$-function, with no need to infer values of classical dynamical variables. In rejecting such "orthodoxy", Einstein suggested an unorthodox alternative according to which

The individual system (before the measurement) has a definite value ...for all variables of the system, and more specifically, that value which is determined by a measurement of this variable. Proceeding from this conception ...the $\psi$-function is no exhaustive description of the real state of the system but an incomplete description. ([15], p. 83)

By accepting Einstein's alternative one can block any inference from entanglement to metaphysical holism or nonseparability. But the "no-go" theorems of Gleason [25], Bell ([2],[3]) and Kochen and Specker [41] have shown that Einstein's alternative is unacceptable: the $\psi$-function or quantum state it represents is not just the source of a probability distribution over possessed values of all dynamical variables. A variety of unorthodox interpretations have come to regard a quantum state as a novel element of physical reality and so reopened the way to metaphysical conclusions that Einstein sought to block. I'll briefly mention some before showing how entangled quantum states provide us with an objective resource in explanatory as well as practical applications of quantum theory even though a quantum state is not an element of physical reality.

\footnotetext{
${ }^{9}$ Rigorously, they can never be localized at a point and the eigenstate-eigenvalue link cannot be applied to either of these states. Physicists commonly assume a vague but stronger principle inferring spatial location from a "suffficiently" localized spatial $\psi$-function. That is why I use the phrase "or something like it". It is interesting to note that the entangled spatial state to which the authors of the EPR argument appealed did not require rigorous localization of component spatial states. But they did apply the eigenstate-eigenvalue link to states without regard for mathematical niceties!
} 
According to what Wallace and Timpson [60] call wave-function realism, the state vector $|\psi\rangle$ is a way of codifying the properties of the physical state. It does this for one-particle quantum mechanics by assigning expectation values to dynamical variables by the usual prescription

$$
\exp A=\langle\psi|\hat{A}| \psi\rangle .
$$

But such an expectation value cannot be understood in the usual way as the mean of a probability distribution for measured values of $A$, since it represents a physical property of an individual system. But what is that system, and how can such a property be understood? The wave function $\psi$ of "an $N$-particle system" is defined at points of a configuration space of $3 N$ dimensions, and $\psi$ may be thought of as a physical field, defined not in ordinary 3-space but in this much higher-dimensional physical space. Both ordinary 3-space and the $N$ "particles" are not parts of the fundamental ontology, but emerge from it given the form of the Hamiltonian defined on $3 N$-dimensional space. In that case $\exp A$ is a property not of any particle or system of particles, but of $3 N$-dimensional space: properties of systems of particles emerge from or are grounded in such fundamental properties of $3 N$-dimensional space along with their bearers. There is no metaphysical holism or nonseparability at the fundamental level where the elementary objects are just points of $3 N$-dimensional space, the $\psi$-function on which fully specifies their properties. But metaphysical holism and nonseparability do emerge at the "manifest" level of particles in ordinary 3 -space.

Wallace and Timpson raise objections against wave-function realism and advocate what they call spacetime state realism as a superior alternative. This takes ordinary 4-dimensional (relativistic) spacetime as physically basic (whether or not it can be reduced to relations between "events"). A density operator (or other mathematical object) defined on each open region of spacetime defines an expectation value of dynamical variables for that region, now understood as properties of the region. If $A, B$ are two such regions then the density operators $\rho_{A}, \rho_{B}$ are the partial traces of $\rho_{A B}$ over the Hilbert spaces $\mathcal{H}_{B}, \mathcal{H}_{A}$ respectively. Since $\rho_{A}, \rho_{B}$ do not determine $\rho_{A B}$, the properties of $A, B$ do not determine those of $A+B$ and so we have metaphysical holism and nonseparability. Indeed, even Weak Separability fails.

Weak Separability: Any physical process occupying spacetime region $\mathrm{R}$ supervenes upon an assignment of qualitative intrinsic physical properties at points of $\mathrm{R}$ and/or in arbitrarily small neighborhoods of those points.

Bohmian mechanics may be regarded as an unorthodox interpretation of non-relativistic quantum mechanics or as an alternative theory. Either way, it treats the wave function as an element of physical reality while denying that it provides a complete description of that reality - a complete description would give the location and velocity of every particle at every moment. Processes 
involving only particles are therefore (at least weakly) spatially and spatiotemporally separable, and the properties of a compound particle system manifest no metaphysical holism even though the motion of a particle depends on the instantaneous positions of all the others. But the wave function does violate spatial and spatiotemporal separability in ordinary 3-space, though not in $3 N$-dimensional configuration space. The wave function does not exhibit metaphysical holism. From the point of view of ordinary 3-space it has no parts, while the properties it may be thought to attribute to any region of $3 N$-dimensional configuration space supervene on the values of $\psi$ at the points composing that region.

There is no need here to consider the metaphysical implications of entanglement in GRW and other non-linear "collapse" theories since these are alternatives to quantum theory rather than unorthodox interpretations of it. Instead I conclude this rapid survey of unorthodox interpretations by noting that on the interactive interpretation I proposed in [26] quantum mechanical entanglement would imply both metaphysical holism and nonseparability. ${ }^{10}$ That interpretation gave the state vector a descriptive role while denying the completeness of this description.

But I have now come to reject not only wave function realism but any view according to which a quantum state is an element of physical reality, whether represented by a wave function, a state vector, a density operator or any other mathematical object. This does not mean quantum states are unreal, subjective, or merely convenient fictions. Quantum states are objective: whether a quantum state assignment is true or false depends on how the world is, no matter what anyone may think. But to assign a quantum state is not to describe a physical system but to do something else, as I'll explain in the next section. A correct understanding of the function of quantum state assignments will show why entanglement has such limited metaphysical significance.

\section{Quantum States as Objective Prescriptions}

Schrödinger [56] entertained the view that a representative ( $\psi$-function) of a quantum state represents a state of knowledge - a catalog of expectations concerning possible future measurement outcomes. This view differs from that suggested by Einstein because the limited knowledge represented by the $\psi$-function concerns not the present condition of the system assigned this state, but the outcome if a measurement of any kind were performed on it. Such a view has been expressed so often over the years that it has earned the right to be considered orthodox. $^{11}$ But it is problematic for at least two reasons: Who are "we", and

\footnotetext{
${ }^{10}$ Indeed this interactive interpretation was what motivated the analysis of holism and nonseparability in my [27], not some more "orthodox" interpretation.

${ }^{11}$ Here are two instances among many.

...the state vector is only a shorthand expression of that part of our information concerning the past of the system which is relevant for predicting (as far as possible) the future behavior thereof. ...the laws of quantum mechanics only furnish
} 
what is it for "us" to observe or measure a system?

A satisfactory account of the nature of quantum states will not mention observers or their activities. How is this possible if a quantum state is a state of knowledge about measurement outcomes? As Schrödinger put it, "it" is namely a sum of knowledge; and knowledge, that no one knows, is none. One can solve this problem and come to understand quantum states by asking not whether a quantum state is a state of knowledge or a state of the world but by asking what function is served by assigning a quantum state. It then becomes clear that the constitutive function of a quantum state assignment is not to describe (the world or "our" knowledge of it) but to prescribe.

To assign a quantum state is to prescribe a state of belief - more specifically, a state represented by an assignment of credences to certain statements about the values of dynamical variables. ${ }^{12}$ To accept quantum theory is to allow one's credences to be guided by this prescription. However it is represented, a quantum state is objective not because it is an element of physical reality, but because it is authoritative. To assign a quantum state is to claim it prescribes the right credences: if this claim is false, then so is that quantum state assignment. Since a statement assigning the correct quantum state to a system is true no matter what anyone may think, Peirce [51] would count this state as real: Leifer [44] would count it as ontic rather than epistemic because it would still exist if all intelligent beings were suddenly wiped out from the universe. But quantum states are neither physical objects nor physical magnitudes. In Bell's [4] terminology, a quantum state is not a beable.

As physically situated, and so epistemically limited, a user of quantum theory can assign quantum states on the basis of what that agent is in a position to know, in order to form reasonable expectations about what that agent is not in a position to know. The information to which an agent has access depends on that agent's physical situation. A single agent may gain access to additional information merely with the passage of time, while spatially separated agents typically have access to different information just because they are in different places. It is not only spatiotemporal location that imposes physical limits on one's knowledge of what has happened to a system. Acquisition of observational knowledge depends on the presence of physical channels capable of conveying that knowledge to the observer, which is why it is so hard to observe dark matter in distant galaxies. ${ }^{13}$

probability connections between results of subsequent observations carried out on a system. ([62], p.166).

...the quantum state of a Qbit or a collection of Qbits is not a property carried by those Qbits, but a way of concisely summarizing everything we know that has happened to them, to enable us to make statistical predictions about the information we might then be able to extract from them. (Mermin [50], p. 109)

\footnotetext{
${ }^{12}$ Here I part company with self-styled quantum Bayesians who take an agent's quantum state to describe her personal state of belief. In section 4 of [30] I contrast both their view and Rovelli's relationism with the present view of quantum states as objective but relational.

${ }^{13}$ In the "paradox" of Wigner's friend it is the absence of such channels that places Wigner (outside the laboratory) in a different agent situation from his friend before he joins him in
} 
We often apply quantum theory not to the actual world but to some simplified or otherwise modified variant of it - to a merely possible world. Clearly the agent applying the model is not located in that merely possible world. But even such applications are from the perspective of some merely hypothetical agent in that world - the perspective of what I will call an agent-situation. All applications of quantum theory are from the perspective of a physicallycharacterized agent-situation. Applications to merely possible worlds make it particularly clear that no agent need occupy such an agent-situation. An agentsituation is characterized, in part, by a space-time region marking the momentary location of a hypothetical agent. It is common to idealize the histories of observers by timelike curves in a relativistic space-time - their world-lines. In this idealization the space-time location of an agent-situation would be a point $p$ on such a curve.

Since no physical processes can convey information faster than light, no agent at $p$ would have observational access to anything outside the past light cone of $p$. So the conditions warranting a quantum state assignment relative to $p$ lie in (or on) the past light cone of $p$. A quantum state assignment to a system relative to $p$ will be a function of everything in the past light cone of $p$ (though much of what happens there will prove irrelevant). But it is important to notice that quantum state assignments relative to $p, q$ may differ: conditions obtaining in the past light cone of $p$ may warrant a different assignment than conditions obtaining in the past light cone of $q$. Since every quantum state assignment is relative to an agent-situation it is misleading to speak of a system's being in a quantum state, as if this expressed a property of that system rather than a relation to the agent-situation from whose perspective it is assigned.

By accepting that the constitutive function of a quantum state assignment is not descriptive but prescriptive we have seen why a quantum state need not be a state of anyone's knowledge, even though it can serve as a reliable source of belief for any agent who happened to occupy the agent-situation from whose perspective it is correctly assigned. But if this has to be understood as belief about measurement outcomes then we have not yet succeeded in excising "the observer" from the story. To do so we will need to examine exactly when, and in what statements, a quantum state prescribes credences.

A few paragraphs back I represented the relevant belief state as a set of credences in certain statements about the values of dynamical variables. I use Lewis's [45] term 'credence' to refer to a minimally rational degree of belief. For an agent's degrees of belief in a set of statements $\left\{A_{i}\right\}$ to be minimally rational is for them to satisfy internal coherence conditions sufficient to prove that they may be represented together by real numbers $0 \leq C r\left(A_{i}\right) \leq 1$ that satisfy a version of the axioms of probability theory (e.g. If $A_{1}, A_{2}$ are incompatible then $\operatorname{Cr}\left(A_{1} \vee\right.$ $\left.\left.A_{2}\right)=\operatorname{Cr}\left(A_{1}\right)+C r\left(A_{2}\right)\right)$. I'll call a statement of the form The value of dynamical variable $M$ on physical system s lies in $\Delta$ a canonical magnitude claim and write it as $M_{s} \in \Delta$. A quantum state assignment exercises its constitutive function when an agent sets credences in canonical magnitude claims equal to their Born

the lab. 
probabilities.

When legitimately applied to the state vector $|\Psi(t)\rangle$ of a system $s$ at time $t$, the Born rule yields probabilities for canonical magnitude claims about the values of dynamical variables $A, B, \ldots$ on $s$

$$
\operatorname{Pr}([A \in \Gamma] \&[B \in \Delta] \& \ldots)=\left\langle\Psi(t)\left|P^{A}(\Gamma) . P^{B}(\Delta) \ldots\right| \Psi(t)\right\rangle \quad \text { (Born rule) }
$$

An application of the Born rule is legitimate only if the projection operators $P^{A}(\Gamma), P^{B}(\Delta), \ldots$ (corresponding to magnitude claims $A \in \Gamma, B \in \Delta, \ldots$ respectively) pairwise commute (e.g. $\left.\left[P^{A}(\Gamma), P^{B}(\Delta)\right]=0\right)$. This condition is satisfied trivially for a single magnitude claim $A \in \Gamma$, which suggests that application of the Born rule prescribes a credence for every magnitude claim $A \in \Gamma$ about $s$ at $t$. But the "no-go" theorems that ruled out Einstein's unorthodox suggestion show that if quantum states assigned to $s$ are represented by vectors in a Hilbert space of 3 or more dimensions, then the Born rule cannot prescribe a set of credences defined over all canonical magnitude claims about $s$-applying the Born rule to any state vector would leave one with degrees of belief that failed to be coherent. ${ }^{14}$

Restrictions on use of the Born rule are needed to ensure its consistent application to a system, but it is unsatisfactory to restrict its use to measurement contexts so that quantum theory becomes merely an algorithm for predicting the outcomes of "measurements" or "observations", whatever these are supposed to be. Bell ([4], p. 215) was quite right to reject the use of such words in a formulation of quantum theory with any pretension to physical precision. There is no such use in the above formulation of the Born rule. Moreover, use of that rule is not restricted to "measurement" contexts: its application is restricted only to canonical magnitude claims with well-defined content. Restrictions on application of the Born rule to magnitude claims about a system $s$ arise naturally from $s$ 's physical circumstances, since which of these claims have well-defined content depends on its environment. My [31] applies an inferentialist view of content to a simple model of decoherence to illustrate the dependence of content on physical environment.

The Born rule may be legitimately applied only when such decoherence has occurred, and then only to those contentful magnitude claims corresponding to projection operators onto subspaces in the relevant pointer basis. Such decoherence is never perfect, and nor is a "pointer basis" precisely determined and perfectly constant. But the advice provided by the Born rule concerning only meaningful magnitude claims privileged by pointer bases in the same narrow neighborhood will be consistent and typically prove reliable: these magnitude claims will typically turn out to be true with relative frequencies closely corresponding to their Born rule probabilities. That is why the evidence

\footnotetext{
${ }^{14}$ Here is another way to see the problem. Fine [1982] showed that the CHSH [1969] inequalities express necessary and sufficient conditions for probability distributions of variable pairs generated by applying the Born rule to a state vector in a Hilbert space of 4 or more dimensions to equal marginals of a joint probability distribution over all canonical magnitude claims about it. Gisin's theorem shows those inequalities are violated by any entangled state of such a system.
} 
warrants acceptance of quantum theory by following its prescription in setting credences concerning magnitude claims about physical systems, whether or not these claims may be taken to report outcomes of measurements.

So a quantum state need represent no observer's knowledge, and the Born rule may be consistently stated and applied with no reference to observation or measurement.

\section{Entanglement's Explanatory Role}

Entanglement is not only a resource to be exploited in technological applications of quantum information and communication but also, I'll argue, an objective resource in explanatory applications of quantum theory.

Practical applications of entanglement depend on two features of the correlations successfully predicted by assignment of entangled quantum states. They are non-classical insofar as they violate Bell inequalities; and they are highly selective. There are, for example, Bell inequalities whose violation by correlations between variables on $A$ and $B$ is incompatible with their violation between corresponding variables on $A$ and $C .{ }^{15}$ Calling entanglement a resource and drawing attention to its potentially important practical applications may suggest that it at least represents a physical relation between systems, irrespective of that relation's metaphysical relevance. But entanglement's practical applications all flow from novel features of the correlations successfully predicted by the assignment of entangled quantum states, and to reap the benefits of an application it is enough to take these to be predictions of outcomes of measurements whose performance is increasingly becoming part of routine laboratory practice.

Whatever their practical usefulness, the correlations successfully predicted by assignment of entangled quantum states have seemed surprising and puzzling. They cry out for explanation while resisting any explanation of a familiar kind. Indeed Bell himself argued that

certain particular correlations, realizable according to quantum mechanics, are locally inexplicable. They cannot be explained, that is to say, without action at a distance. ([4], p. 152)

These and similar correlations have now been realized many times ${ }^{16}$. Some have been content to leave the puzzling correlations unexplained. But quantum theory is generally regarded as our most powerful explanatory theory, enabling us to understand all kinds of physical phenomena that eluded the grasp of classical physics. Why can't quantum theory explain the correlations it so successfully predicts?

Some (including my former [28] self!) have appealed to ideas of nonseparability or holism as the key to their quantum explanation with no action

\footnotetext{
${ }^{15}$ Seevinck [58] provides examples in a nice review of work relating the monogamy of entanglement to the shareability of non-classical correlations.

${ }^{16}$ Giustina et al. [23] report a recent advance on seminal previous experiments to which they refer.
} 
at a distance. If successful, this might support an inference to metaphysical nonseparability and/or holism as required by the best explanation of quantum correlations including those violating Bell inequalities. But such appeals have not been incorporated into a defensible, Lorentz-invariant interpretation of quantum theory ([10], [48]), and nor is it clear how they can evade the logic of Bell's argument ([35]). No inference to metaphysical conclusions as required by the best explanation of the puzzling quantum correlations is plausible in the light of current knowledge. Such an argument would require either successful development of a novel interpretation of quantum theory or construction of a new physical theory. In either case it would be necessary to point to a loophole in Bell's argument.

Everettians can respond to Bell's argument by denying its assumption that a measurement always has a unique outcome, maintaining on the contrary that measurements of quantum "observables" generally have different outcomes in different "branch-worlds". Without this assumption Bell's argument does not go through, whether or not the world is fundamentally separable. So for an Everettian it is the multiplicity of "branch-worlds" that is key to the quantum explanation of non-classical correlations with no action at a distance, not the truth of metaphysical nonseparability or holism.

Maudlin ([47]) argued that space-like (superluminal) influences must be present in experiments verifying them: Price ([52]), on the other hand, suggested their results might be explained by retrocausal influences. One way for a measurement on one photon in an EPR-Bohm pair to influence the result of a spacelike separated measurement on the other photon would be through "collapsing its quantum state"—just the kind of "spooky" action at a distance Einstein rejected. Though problematic, such nonstandard causal accounts need appeal to no metaphysical holism or nonseparability.

In fact "single world" quantum theory may be applied to explain EPRBohm correlations with no superluminal or retro- causation, no quantum state collapse and no metaphysical holism or nonseparability. To prepare the way for my later remarks on metaphysics I'll give a quick sketch of the explanation here. ${ }^{17}$ The relativization of quantum state assignments to agent-situation is key to this explanation. An entangled state $\Phi^{+}=1 / \sqrt{ } 2(|H H\rangle+|V V\rangle)$ may be correctly assigned to a photon pair relative to an agent-situation with location $p$ in the past light cone of either of the space-like separated polarization measurement events: it is warranted by whatever physical conditions (in the past light cone of $p$ ) the experimenters used to prepare that state, such as parametric down-conversion of laser light by passage through a non-linear crystal.

Because of decoherence at the detectors, the Born rule is legitimately applied to this state to yield an equal chance of either outcome of any linear polarization measurement at either detector, but chance $\cos ^{2} \theta$ that the two detectors will yield the same outcome for linear polarization measurements with respect to axes inclined at an angle $\theta$ to one another. It is important to stress

\footnotetext{
${ }^{17}$ For a fuller account see my [34]. Note that both the sketch, section 9.1, and much of section 10 depend on the view of quantum states presented in section 7 (see also [30]).
} 
that these chances are also relative to agent-situation with location $p$. For anyone who accepts quantum theory, they give the objectively correct advice to one located at $p$ about how firmly to believe the corresponding outcome(s) will be recorded. Thus anyone warranted in assigning state $\Phi^{+}$is able to form reliable expectations about correlations between outcomes of polarization measurements, as expressed by magnitude claims about the detectors.

Consider instead an agent situation with location $q$ in the future light cone of recording event $\mathbf{1}$ but not of recording event $\mathbf{2}$. Relative to $q$, the correct quantum polarization state to assign to the distant photon in the past light cone of $\mathbf{2}$ depends on the outcome at $\mathbf{1}$ : suppose it is $V_{a}$, where linear polarization at $\mathbf{1}$ was measured with respect to axis $\theta_{a}$. Then $\left|V_{a}\right\rangle$ is the correct quantum state to assign to the distant photon, relative to agent situation with location $q$. This is not because "the" quantum state of the pair has collapsed: the correct quantum state to assign relative to agent-situation with location $p$ is still represented by density operator $\frac{1}{2} \hat{1}$ - the reduced state of $\Phi^{+}$. The correct state relative to agent situation with location $q$ is $\left|V_{a}\right\rangle$ because the outcome at $\mathbf{1}$ is in the past light cone of $q$, and so counts as an additional accessible condition thus warranting the assignment of state $\left|V_{a}\right\rangle$ relative to $q$. Relative to agent situation with location $q$ the chance of outcome $V_{b}$ at $\mathbf{2}$ is $\cos ^{2}\left(\theta_{a}-\theta_{b}\right)$, as follows from the legitimate application of the Born rule to state $\left|V_{a}\right\rangle$.

These applications of quantum theory explain the patterns of correlation that are taken to violate $\mathrm{CHSH}$ inequalities by showing that they were to be expected and what they depend on. Both individual outcome events and the event of their joint occurrence depend causally on the physical conditions warranting assignment of $\Phi^{+}$since an agent could affect the chances of these events by modifying those conditions. But there is no causal dependence of one outcome event on the other, since no-one who accepts quantum theory can countenance the possibility of an agent's modifying either outcome while keeping fixed both detectors' settings and the conditions warranting assignment of $\Phi^{+}$. Nor do the detector settings have any retrocausal influence on events acknowledged by quantum theory.

This explanation of Bell's "inexplicable" correlations appeals to no metaphysical principles such as holism or nonseparability. But the way it treats causation, chance, modality and explanation itself conveys important lessons for metaphysics. The last two sections will make these lessons explicit. But first I want to clear up two issues raised by talk of grounding. First, in previous publications (including [33]) I talked that way about the conditions warranting assignment of a quantum state, so suggesting yet another application of a concept currently fashionable among metaphysicians. Secondly, this concept was recently applied (by Ismael and Schaffer [39]) in connection with the nonseparability that some have thought to lie behind the phenomenon of quantum entanglement. But neither application is warranted if the function of a quantum state assignment is not to describe the world but to prescribe belief about it. 


\section{No Grounds for Entanglement or Nonsepara- bility}

\subsection{Backing is not grounding}

I said that a quantum state is objective not because it is an element of physical reality, but because it is authoritative, and that to assign a quantum state is to claim it prescribes the right credences, relative to an agent-situation. But what makes one set of credences right and another wrong? What is it about such situations that provides the backing for assignment of one quantum state rather than another? Without an answer to this question, assigning a quantum state and basing one's expectations on probabilities generated by the Born rule would be unjustified: following the quantum state's prescription would be like basing one's beliefs on the pronouncements of an oracle with a good track record.

One may be warranted in assigning a particular quantum state while ignorant of the physical conditions that back it, much as you may be warranted in following a doctor's prescription even though you don't know what makes the prescription work. But there is an important disanalogy. It is following the prescription that brings about recovery, whereas using the assigned quantum state to set one's expectations does not bring about events that fulfill them. One is warranted in basing one's expectations on assignment of a particular quantum state because whatever physical conditions provide the backing for that assignment are reliably correlated with the statistics it leads one to expect.

Experimental physicists talk of preparing quantum states. Such talk may be understood as a way of specifying the physical conditions warranting the resulting quantum state assignment. For example, to prepare a beam of $\mathrm{C}_{60}$ molecules in a pure (center of mass) quantum state effectively certain to yield value $p_{x}$ in a measurement of $x$-component of momentum, one can pass the beam through spinning disks that pass only molecules with the corresponding velocity: to prepare a collection of rubidium atoms in a state corresponding to a Bose-Einstein condensate, one inserts a few into a high vacuum, cools them to within 1 millionth of a degree of absolute zero using carefully designed laser beams and evaporative cooling and traps them with magnetic fields. ${ }^{18}$

But there are two reasons why we can't rest content with such a way of specifying the physical conditions warranting a quantum state assignment. First, if quantum theory is to be applied outside the laboratory it must be possible to assign quantum states to systems that no-one has prepared. More fundamentally, this kind of specification makes free use of the language of classical physics, but why are we entitled to use such language in specifying the conditions warranting a particular quantum state assignment?

Fortunately, we can appeal once more to quantum decoherence to quiet these worries. In section $7 \mathrm{I}$ appealed to decoherence to show how to state and apply the Born rule with no mention of measurement. But models of

\footnotetext{
${ }^{18}$ See http://www.colorado.edu/physics/2000/bec/how_its_made.html for a more complete elementary description.
} 
decoherence may be much more widely applied to provide reassurance that a host of magnitude claims have well defined empirical content. Some of these truly state the conditions warranting assignment of quantum states. What determine the correct quantum state to assign are the empirically significant and true magnitude claims that state its backing conditions. The quantum state assignment supervenes on these statements. The same thing applies to an enormous number of statements about the physical world and not only those stated in the language of classical physics, such as the statement that a BoseEinstein condensate was first prepared in 1995.

A quantum state assignment is objective because it is relative to the physical, not merely epistemic, situation of an actual or hypothetical agent. However this physical situation is described, it is true, empirically significant, magnitude claims that make the description correct-importantly including claims about the location of such an agent. In the previous section we saw how Alice and Bob each correctly assigned different quantum states at the same time because they were in different places. An agent's physical situation may influence what information is physically accessible, so the ideal epistemic state of an agent is also relative to the agent's physical situation. But this introduces no element of subjectivity into quantum states or Born probabilities.

A true quantum state assignment supervenes on its backing conditions. It is tempting to conclude that these conditions ground its truth. Unlike quantum state assignments, backing conditions describe the physical world. They may be expressed by magnitude claims - claims about elements of physical reality. Unlike the quantum state, these magnitudes are naturally thought of as beables - even local beables. Backing conditions don't just provide a supervenience base for quantum state assignments, they appear to ground them: as an asymmetric relation, grounding (unlike supervenience) seems to capture the priority of backing conditions to the quantum state assignments they back.

However, grounding is supposed to be a metaphysical relation, while closer inspection shows backing conditions to be merely epistemically prior to the quantum state assignments they back.

Their epistemic priority is clear. While conditions backing assignment of a quantum state to a system are open to observation (however indirect), the state they back is not. Suppose one knows nothing about the correct quantum state to assign to an individual system. No single observation on it can reliably disclose its state. Repeated observations are no better, since observing a system typically irreparably disturbs its quantum state. So even if a system's wholly unknown quantum state could be ascertained by a single observation, this finding could not be checked in further observations, either by the original observer or by others. To come to know the quantum state of a system one must have access to information about it or systems related to it. This must include information expressible in magnitude claims since this is the only objective information observation can supply.

But the epistemic priority of backing conditions expressible as magnitude claims does not make them metaphysically prior to the quantum state assignments they warrant. Quantum states not only prescribe credences in sig- 
nificant magnitude claims: through the application of models of decoherence, they also provide a check on that significance. By modulating the content of a magnitude claim, the quantum state in an appropriate model of environmental decoherence may be thought to control what fact would correspond to the truth of that claim in that environment. This makes such quantum states appear semantically or pragmatically prior to the magnitude claims whose content they control. But of course the quantum states appealed to in a model of decoherence must themselves be warranted by other magnitude claims about that environment: and these states don't so much control as certify the content of magnitude claims about the original system.

This subtle interplay between magnitude claims and assignment of quantum states is not adequately captured by saying that the former ground the latter. On the contrary, it weakens the case for taking magnitude claims as providing a supervenience base of independent physical facts. While a magnitude claim about a system may still be taken to attribute a value to a local beable, the fact that the content of that claim depends on the system's environment introduces an element of holism that is not simply attributable to entanglement. There is a sense in which the physical facts are not wholly independent of one another, whether or not collectively they ground all other facts.

Moreover, physical property holism is not wholy absent from this picture. There are circumstances in which environmental decoherence renders significant claims about values of magnitudes on compound systems but not on their component subsystems. For example, in the large molecule interference experiment of Juffman et al. [40] the position of a $\mathrm{C}_{60}$ molecule's center of mass is rendered determinate by interaction with the screen to which it adheres, but this property of the molecule does not supervene on the positions or any other properties or relations of its component atoms - indeed, claims about just where those atoms are lack empirical significance.

\subsection{Quantum connection by common causes, not grounds}

While not adopting any particular interpretation of quantum theory, Ismael and Schaffer [39] argue that it counts in favor of such an interpretation that it provide for what they call a Common Ground explanation of entanglementunderstood via Nonseparability, in ways which lead to Quantum Connection. Here's what they mean by the italicized expressions.

Nonseparability: Entities $a$ and $b$ are nonseparable if and only if fixing the intrinsic state of $a$, the intrinsic state of $b$ and the spatial relations between $a$ and $b$ fails to fix the intrinsic state of $a+b$.

This is almost the denial of what I earlier called Spatial Separability: it differs only in speaking of two entities rather than any number of systems.

Quantum Connection: In a nonseparable quantum system, nonidentical events $e_{a}$ and $e_{b}$ are modally connected. 
Common Ground: If non-identical events $e_{a}$ and $e_{b}$ are modally (but not causally) connected then $e_{a}$ and $e_{b}$ are grounding-connected (/non-distinct), in that either (i) $e_{a}$ grounds $e_{b}$, or (ii) $e_{b}$ grounds $e_{a}$, or (iii) $e_{a}$ and $e_{b}$ are joint results of some common ground $c$.

I have changed their notation in these last two principles to avoid the suggestion that $a, b$ in the first principle must be understood as events (rather than systems or other entities).

Before we get to ground, I have three remarks about entanglement and Nonseparability. First, in one orthodox view (as elaborated in section 7), entanglement is not a physical relation between systems that is in need of explanation. Rather, by assigning entangled quantum states we can use quantum theory to explain otherwise inexplicable patterns of correlation. Second, Nonseparability is neither a way of understanding entanglement nor an explanation of the non-classical correlations correctly predicted by assignment of entangled states. Finally, to assign an entangled quantum state is not to describe the intrinsic state of a system: neither it nor its subsystems need be describable by empirically significant magnitude claims, and even if they are it is not clear that these specify their intrinsic states.

Quantum Connection ascribes a modal connection to certain events involving a nonseparable quantum system. Passing over the reasons just given for doubting that there are any nonseparable quantum systems, it is appropriate to ask what are the events $e_{a}$ and $e_{b}$ and what it is for them to be modally connected. Ismael and Schaffer consider a familiar EPR-Bohm pair, say the hydrogen atoms formed by dissociation of a hydrogen molecule assigned state (7). They believe the pair of atoms $a$ and $b$ are nonseparable, and consider events $e_{a}$ and $e_{b}$ in which $a, b$ respectively "measure out at", one with spin up, the other with spin down with respect to the $x$-axis. What modal connection between $e_{a}$ and $e_{b}$ is supposed to follow from ascription of the spin state (6) to $a+b$ ?

Modality is often associated with necessity and possibility, so one might take Quantum Connection to assert that neither of $e_{a}, e_{b}$ can occur without the other (even though these events are spatially separated). But this is not plausible. Suppose that $a$ is measured somewhere and $b$ is due to be measured at some distant location - in Leiden and Rio respectively in my fanciful illustration - but the Rio apparatus doesn't work! Then $e_{a}$ occurs ( $a$ "measures out at" $x$-spin up, say), but $e_{b}$ does not $-b$ never "measures out". ${ }^{19}$ The "collapse" postulate that goes along with Einstein's "orthodox" view of the descriptive completeness of the state vector implies (in conjunction with the eigenstate-eigenvalue link) that $a$ has $x$-spin up immediately after "measuring out at" $x$-spin up, or $x$-spin down immediately after "measuring out at" $x$-spin down, and similarly for $b$ but with the opposite outcome. If one accepts such an interpretation, one can take $e_{a}, e_{b}$ to be events that happen to $a, b$ respectively at which each acquires an intrinsic property it had previously lacked. The modal connection would then

\footnotetext{
${ }^{19}$ This would happen most of the time in any realistic experiment, since detector efficiencies are usually well below $100 \%$.
} 
be that each acquires an $x$-spin property if and only if the other acquires the opposite property - one up, the other down. Most importantly, as soon as either $a$ or $b$ is measured, the other atom acquires the opposite property - it doesn't have to wait until it reaches its detector before doing so.

This appears to restore a necessary connection, but only at the price of introducing a preferred notion of simultaneity. That is just one of the problems associated with such an "orthodox" interpretation that takes a state vector to provide a complete description of the system to which it is assigned. As shown in the last section, no such problem arises if quantum states are understood along the lines of section 7. A measurement in Leiden has no immediate effect in Rio, and neither measurement need result in the atom acquiring the property it records: in analogous measurements of photon polarization the EPR-Bohm photons are simply absorbed into the detectors. My main point here is that whether there is any modally necessary connection requiring explanation here is highly interpretation dependent, and that there is interpretative pressure in favor of a view of quantum states that denies that there is such a connection.

Alternatively, one might take a relation of mutual counterfactual dependence between any pair of events $e_{a}, e_{b}$ at which Alice and Bob both actually "measure out" to constitute a modal connection between these events, even though either might have occurred without the other given the quantum state assigned to $a+b .^{20}$ In evaluating such a counterfactual it is natural to hold fixed that each of $a, b$ "measured out", whether as $x$-spin up or $x$-spin down. Assignment of spin-state (6) then justifies one in assigning credence 1 to this counterfactual. In that sense quantum theory supports the counterfactual, even though it is implied by no quantum law (neither a dynamical law nor a law of coexistence like the perfect gas law).

If there were a modal quantum connection, could it be explained by Common Ground? In the case of spacelike separated measurements on EPRBohm pairs, considerations of spacetime symmetry militate against an explanation of types (i) and (ii), thereby favoring a type (iii) explanation. Ismael and Schaffer consider a type (iii) common ground explanation preferable. But what is $c$ ? It's just $a+b$ :

Singlet (i.e. (6)) is a property of the whole $a+b$ system. This property of the whole system can explain the behavior of its components ( $a$ as well as $b$ ) because the whole system grounds the components.

I see two problems with this proposal. The first problem is that a variety of interpretations of quantum theory deny that Singlet (i.e. (6)) is a property of the whole $a+b$ system. Besides the orthodox interpretation described and developed in section 7 these include Einstein's unorthodox alternative, Wallace and Timpson's spacetime state realism, and Bohmian mechanics (all discussed in section 6). Ismael and Schaffer may acknowledge this while continuing to maintain that it counts in favor of an interpretation of quantum

\footnotetext{
${ }^{20}$ Correspondence with Ismael confirms this is indeed the kind of modal connection intended in [39].
} 
theory that it permit this common ground explanation of Quantum Connection (and more generally of non-localized correlations that violate Bell inequalities) without running afoul of Bell's theorem (their italics).

The point of a common ground story is to give up locality (understood in terms of a "screening off" condition) without positing any hidden variables, and without positing any causal connection.

But interpretations accepting quantum state assignments as specifying properties of the systems to which they are assigned (in particular (6) as specifying a property of the whole $a+b$ system) must provide a Lorentz invariant account of how such properties change on measurement (for example, as $a, b$ each "measure out"). Myrvold [49] provides a Lorentz invariant framework into which a collapse theory may be inserted, but such theories are alternatives to rather than interpretations of, quantum theory. His earlier paper [48] showed how hard it is to develop a Lorentz invariant modal interpretation in which a quantum state specifies nonseparable properties of systems to which it is assigned. I know, because I tried [28]!

Ismael and Schaffer acknowledge in a footnote that they freely move back and forth between object talk and event talk. This suggests that an alternative way to implement the Common Ground strategy would be to take as $c$ not $a+b$ but a compound event $e_{a} \uplus e_{b}$ whose occurrence is constituted by the joint occurrence of $e_{a}$ and $e_{b}$. This implementation arguably relieves any tension with Lorentz invariance but faces other challenges.

That $e_{a}$ and $e_{b}$ are modally connected through their common ground $e_{a} \uplus e_{b}$ is not much of an explanation if $e_{a} \uplus e_{b}$ remains unaccounted for. In the following section I'll show that, if interpreted along the lines of section 7 , quantum theory can explain not only $e_{a} \uplus e_{b}$ but also $e_{a}, e_{b}$ themselves as effects of state (6)'s backing conditions. This explanation by a common cause that fails to screen off its localized effects renders superfluous any common ground explanation of the counterfactual connection between $e_{a}$ and $e_{b}$.

Moreover, in case $e_{a}, e_{b}$ are not space-like but time-like separated $e_{a} \uplus e_{b}$ is a temporally non-localized event. When $e_{a}$ but not $e_{b}$ has happened, $e_{a}$ would, on this proposal, be grounded in an event $\left(e_{a} \uplus e_{b}\right)$ that has not yet wholly occurred. Even those willing to entertain the idea of grounding as a priority relation may be unwilling to allow such a metaphysical possibility.

\section{Quantum Fool's Gold}

Quantum entanglement has been taken to raise the prospect of some kind of metaphysical holism or nonseparability. If to assign a quantum state is not to describe physical reality, but to offer objective prescriptions concerning the significance and credibility of beliefs about it, then that prospect appears bleak. But that is not to say entanglement is without philosophical interest.

On the contrary, one can learn a lot about chance, causation and explanation by reflecting on the role of entanglement in explaining non-classical 
correlations like those Bell took to be inexplicable without action at a distance. Since these concepts have provided the focus of much metaphysical discussion (not only among professional philosophers) entanglement does have implications for metaphysics. But I take these to be negative implications for the practice of metaphysics rather than positive metaphysical conclusions. The explanation of non-local correlations requires no metaphysical holism. But it should prompt philosophers to question whether a philosophical investigation of chance, causation and explanation is best pursued by the methods of contemporary analytic metaphysics. Prospectors for gold can be disappointed to find only fool's gold. But small amounts of precious metals can be extracted from iron pyrite deposits. That metaphor prompted the title of this section.

In section 8 I claimed that quantum theory may be applied to explain Bell's particular correlations by showing that they were to be expected and what they depend on. To amplify this brief summary I'll begin by considering exactly what is to be explained. Here it is important to distinguish between statistical correlations exhibited in actual experiments (or elsewhere in nature) and the probabilistic phenomenon these may be taken to manifest. What is a probabilistic phenomenon?

Experimenters often plot a smooth curve through data points to indicate the relationship between values of the plotted magnitudes, such as the extension of a spring when different small weights are suspended from it. According to Hooke's law this is a linear relationship. Data points from actual experiments manifest Hooke's law: they do not constitute it. It is a fact about the data from many properly conducted experiments that they may be well modeled by Hooke's law. When we give an explanation of Hooke's law we explain this fact, not actual data obtained in experiments on springs. This example is typical: when a phenomenon is explained, the explanandum is not the actual data that manifest it, but the fact that those data may be well modeled by a generalisation that abstracts from the complex details of the processes that produce data in particular circumstances, and idealizes and extrapolates from such data. Most interesting scientific explanations are not of particular events but of such general phenomena.

If the association between data specifying values of magnitudes is merely statistical, one cannot plot a single smooth curve to indicate any fixed relationship between them. Instead one can often give a probabilistic model. Such data are often the result of multiple complex processes whose details we can neither predict nor control. Casinos depend both on the unpredictability of the outcome of any given spin and on a probabilistic model of the outcome statistics of large numbers of spins that assigns equal probability to the ball landing in each sector of the wheel. It is because the outcomes of spins of a roulette wheel and tosses of a biased coin are best modeled probabilistically that I call them probabilistic phenomena.

In explaining a probabilistic phenomenon one does not give a causal explanation of individual events whose statistical distribution one takes to manifest it. Instead, one shows how the probabilities defining the phenomenon are themselves just particular instances of a more general class of probabilities exhibited 
by a wide range of other phenomena. All phenomena in this class are thereby unified by the probabilistic theory used to model them. Viewed as a probabilistic phenomenon, Bell's particular correlations are explained by showing how they may be modeled by quantum theory's Born probabilities. To exhibit some probabilities as an instance of a wider class of probabilities is not to explain them causally: probabilities are not events, and a probabilistic phenomenon is not a compilation of actual events. Indeed, when Bell was writing (in 1981) no actual events were known to manifest the particular correlations of atom pairs he took to defy explanation without action at a distance.

Woodward ([63], p. 219) has remarked on a common feature of causal and non-causal explanations:

[. . .] the common element in many forms of explanation, both causal and non-causal, is that they must answer what-if-things-hadbeen-different questions [ $w$-questions].

The quantum explanation of Bell's correlations as a probabilistic phenomenon can answer many $w$-questions, such as what probabilistic phenomenon would be manifested by a different entangled state, or by different particles, or if the same state had been prepared by an entanglement-swapping operation, or if a particle had passsed through a magnetic field prior to detection. By doing so it unifies all the other phenomena: it shows what they have in common and thereby helps us better to understand them all. These counterfactual answers to $w$-questions also show what Bell's correlations depend on.

But causal considerations are not irrelevant here, since there are also dependency relations among events in any instance that may contribute to a manifestation of the phenomenon. So showing that a probabilistic phenomenon is a case of a more general probabilistic phenomenon provides a satisfactory explanation only if these events exhibit the right kind of dependency relations. Indeed, it is just such relations that Bell maintains must exhibit problematic causal dependencies in any explanation of the correlations. So it is important to show why the quantum explanation sketched in section 8 involves no instantaneous action at a distance. To see that it does not we need to understand how Born probabilities are applied to yield chances, and how chances are related to causal dependence.

By chance I mean the definite, single-case probability of an individual event such as rain tomorrow in Tucson. As in this example, its chance depends on when the event occurs - afterwards, it is always 0 or 1: and it may vary up until that time as history unfolds. Chance is important because of its conceptual connections to belief and action. The chance of $e$ provides an agent's best guide to how strongly to believe that $e$ occurs, when not in a position to be certain that it does. ${ }^{21}$ And the comparison between $e$ 's chances according as (s)he does or does not do $D$ are critical in the agent's decision about whether to do $D$. These connections explain why the chance of an event defaults to 0 or 1

\footnotetext{
${ }^{21}$ I use the "tenseless present" rather than the more idiomatic future tense here for reasons that will soon become clear.
} 
when the agent is in a position to be certain about it - typically, after it does or doesn't occur.

Probabilistic theories may be useful guides to the chances of events, but what they directly yield are not chances but general probabilities of the form $\operatorname{Pr}_{C}(E)$ for an event of type $E$ relative to reference class $C$. To apply such a general probability to yield the chance of $e$, you need to specify the type $E$ of $e$ and also the reference class $C$. A probabilistic theory may offer alternative specifications when applied to determine the chance of $e$, in which case it becomes necessary to choose the appropriate specifications. Actuarial tables may be helpful when estimating the chance that you will live to be 100 , but you differ in all kinds of ways from every individual whose death figures in those tables. What you want is the most complete available specification of your situation: this may include much irrelevant information, but it's not necessary to exclude this since it won't affect the chance anyway. In Minkowski space-time, the conceptual connection between chance $(C h)$ and the degree of belief $(\mathrm{Cr})$ it prescribes is captured in this version of David Lewis's Principal Principle that implicitly defines chance: ${ }^{22}$

The chance of $e$ at $p$, conditional on any information $I_{p}$ about the contents of $p$ 's past light cone satisfies: $C r_{p}\left(e / I_{p}\right)={ }_{d f} C h_{p}(e)$.

Suppose that in the scenario of section 8 Alice repeatedly measures the polarization of a photon $a$ in a pair and Bob measures the polarization of paired photon $b$ with respect to the same axis $\left(\theta_{a}=\theta_{b}\right)$, where these measurements are always spacelike separated but Alice's occurs slightly later in their laboratory frame. Now consider an agent who accepts quantum theory and wishes to determine the chance of the event $e_{A}$ that the next photon detected by Alice registers as of polarization type $V($ ertical $)$. Assuming that the state $\Phi^{+}$was prepared and decisions to measure polarization with respect to this axis made long before, the agent is also in a position to be certain what these were. The agent is then in a position to use the Born rule to determine the chance of $e_{A}$. But that chance must be relativized not just to a time, but (relativistically) to a space-time point.

Let $p$ be a point just in the past light cone of Alice's measurement in region 1 and $p^{\prime}$ be just in the past light cone of Bob's measurement in region 2 , while $q$ is just in region 2's future light cone, simultaneous in the laboratory frame with $p$. Suppose Bob's photon registers as vertically polarized and we wish to apply the Born rule to find the chance that Alice's will also. Consider an event $e_{A}$ of the next photon detected in $\mathbf{1}$ 's future light cone registering as vertically polarized: i.e. that $e_{A}$ is of type $V$. The specification of the reference class at least includes the state and the axes of polarization measurement: but for $C h_{q}\left(e_{A}\right)$ we further need to specify a type $V$ outcome in region $\mathbf{2}$. Hence

\footnotetext{
${ }^{22}$ See Ismael [38]. I have slightly altered her notation to avoid conflict with my own. Here ' $e$ ' ambiguously denotes both an event and the proposition that it occurs. $\mathrm{Cr}$ stands for credence: an agent's degree of belief in a proposition, represented on a scale from 0 to 1 and required to conform to the standard axioms of probability.
} 


$$
\begin{gathered}
C h_{p}\left(e_{A}\right)=\operatorname{Pr}_{\Phi^{+}}\left(V_{A}\right)=\frac{1}{2}, \\
C h_{q}\left(e_{A}\right)=P r_{\Phi+}\left(V_{A} \mid V_{B}\right) \equiv \frac{P r_{\Phi^{+}}\left(V_{A}, V_{B}\right)}{P r_{\Phi^{+}}\left(V_{B}\right)}=\frac{\frac{1}{2}}{\frac{1}{2}}=1 .
\end{gathered}
$$

Any agent who accepts quantum theory and is (momentarily) located at space-time point $x$ should match credence in $e_{A}$ to $C h_{x}\left(e_{A}\right)$ because it is precisely the role of chance to reflect the epistemic bearing of all information accessible at $x$ on facts not so accessible, and to accept quantum theory is to treat it as an expert when assessing the chances. This is so whether or not an agent is actually located at $x$-fortunately, since it is obviously a gross idealization to locate the epistemic deliberations of a physically situated agent at a space-time point! A hypothetical agent located at $q$ in the forward light cone of region $\mathbf{2}$ (but not $\mathbf{1}$ ) has access to the additional information that the outcome in $\mathbf{2}$ is of type $V$ : so the reference class used to infer the chance of $e_{A}$ at $q$ from the Born rule should include that information. That is why $C h_{q}\left(e_{A}\right)$ is determined by the conditional Born probability $\operatorname{Pr}_{\Phi^{+}}\left(V_{A} \mid V_{B}\right)$ but $C h_{p}\left(e_{A}\right)$ is determined by the unconditional Born probability $\operatorname{Pr}_{\Phi^{+}}\left(V_{A}\right)$.

In this case application of the Born rule yields the chances $C h_{p}\left(e_{A}\right)$, $C h_{p^{\prime}}\left(e_{B}\right)=\frac{1}{2}$; but $C h_{q}\left(e_{A}\right)=1$. Bell $([2004$, pp. 240-41] $)$ says

Each of the counters considered separately has on each repetition of the experiment a $50 \%$ chance of saying 'yes'.

After noting that quantum theory here requires a perfect correlation between the outcomes in $\mathbf{1}, \mathbf{2}$, he continues

So specification of the result on one side permits a $100 \%$ confident prediction of the previously totally uncertain result on the other side.

But only for a hypothetical agent whose world-line has entered the future light cone of $\mathbf{2}$ near $q$ is it true that specification of the result in $\mathbf{2}$ permits a $100 \%$ confident prediction of the previously totally uncertain result on the other side. A hypothetical agent at $p$ is not in a position to make a $100 \%$ confident prediction. For such an agent the result in $\mathbf{1}$ remains totally uncertain: what happens in $\mathbf{2}$ makes no difference to what ( $\mathrm{s}$ )he should believe, since region $\mathbf{2}$ is outside the past light cone of $p$. That is why it is $C h_{p}\left(e_{A}\right)$, not $C h_{q}\left(e_{A}\right)$, that says what is certain at $p$. Newtonian absolute time fostered the illusion of the occurrence of future events becoming certain everywhere at the same timewhen they occur if not sooner. Relativity requires certainty, like chance, to be relativized to space-time points - idealized locations of hypothetical knowers.

In one traditional view the past (unlike the future) is fixed, settled or metaphysically certain. Apart from its other problems, this view is hard to formulate in a relativistic spacetime. If one identifies the past with everything earlier than now (as defined by the time of some global frame) then what past events are fixed depends on an arbitrary choice of that frame. If one identifies 
the past with the past light cone, then fixity is relative to the spacetime point whose past light cone it is. Certainty, unlike fixity, represents an epistemic ideal for a physically situated knower: so it has the flexibility required to withstand relativization to potential spacetime locations of a hypothetical, idealized knower.

Bell seemed to view chances as localized physical magnitudes. But quantum theory teaches us that chances are not localized physical propensities whose actualization may produce an effect. Maudlin says what he means by calling probabilities objective:

...there could be probabilities that arise from fundamental physics, probabilities that attach to actual or possible events in virtue solely of their physical description and independent of the existence of cognizers. These are what I mean by objective probabilities. ([1], p. 294)

Although quantum chances do attach to actual or possible events, they are not objective in this sense. As we saw, the chance of outcome $e_{A}$ does not attach to it in virtue solely of its physical description: the chances of $e_{A}$ attach also in virtue of its space-time relations to different space-time locations. Each such location offers the epistemic perspective of a situated agent, even in a world with no such agents. The existence of these chances is independent of the existence of cognizers. But it is only because we are not merely cognizers but physically situated agents that we have needed to develop a concept of chance tailored to our needs as informationally deprived agents. Quantum chance admirably meets those needs: an omniscient God could describe and understand the physical world without it. While they are neither physical entities nor physical magnitudes, quantum chances are objective in a different sense. They supply an objective prescription for the ideal credences of a hypothetical agent in any space-time location. Anyone who accepts quantum theory is committed to following that prescription.

$C h_{q}\left(e_{A}\right)$ depends counterfactually on the polarization measurement in 2 and also on its outcome: had either the outcome or the measurement axis been different, $C h_{q}\left(e_{A}\right)$ would have been different. Don't such counterfactual differences in $C h_{q}\left(e_{A}\right)$ amount to causal dependence between space-like separated events, constituting instantaneous action at a distance? No, for two reasons.

While $C h_{q}\left(e_{A}\right)$ would be different in each of these counterfactual scenarios, in none of them would $C h_{p}\left(e_{A}\right)$ differ from $\frac{1}{2}$, so the "local" chance of $e_{A}$ is insensitive to all such counterfactual variations in what happens in $\mathbf{2}$. If one wishes to infer causal from counterfactual dependence of "the" chance of a result in $\mathbf{1}$ on what happens in $\mathbf{2}$, then only one of two relevant candidates for "the" chance displays such counterfactual dependence. For those who think of chance as itself a kind of indeterministic cause - a localized physical propensity whose actualization may produce an effect- $C h_{p}\left(e_{A}\right)$ seems better qualified for the title of "the" chance of $e_{A}$ than $C h_{q}\left(e_{A}\right) .{ }^{23}$

${ }^{23}$ Friederich makes a similar point in [20]. 
The role of chance in decision provides a more important reason. Just as the chance of $e$ tells you everything you need to know to figure out how strongly to believe $e$, the causal dependence of $e$ on $d$ tells you everything you need to know about $e$ and $d$ when deciding whether to do $d$ (assuming you are not indifferent about $e$ ). As Huw Price [54] put it, "causal dependence should be regarded as an analyst-expert about the conditional credences required by an evidential decision maker".

Consider the situation of a hypothetical agent Bob at $p^{\prime}$ deciding whether to act by affecting what happens in $\mathbf{2}$ to try to get outcome $e_{A}$ in $\mathbf{1}$. Bob can choose not to measure anything, he can choose to measure polarization with respect to any axis, or he can turn on some local interaction. No-signalling theorems, going back to Eberhard [13], Bell ([4], pp. 237-8) show why none of these actions would alter Bob's estimated value of $C h_{q}\left(e_{A}\right) .^{24}$

But what if Bob had simply arranged for the measurement in $\mathbf{2}$ to have had the opposite outcome $\bar{e}_{B}$ (an event of type $H$ (orizontal) rather than $V($ ertical $))$ ? Then $C h_{q}\left(e_{A}\right)$ would have been 0 instead of 1 . No-one who accepts quantum theory can countenance this counterfactual scenario. The Born rule implies that $\operatorname{Pr}_{\Phi^{+}}\left(H_{B}\right)=\frac{1}{2}$, and anyone who accepts quantum theory accepts the implication that $C h_{p^{\prime}}\left(\bar{e}_{B}\right)=\frac{1}{2}$. So anyone who accepts quantum theory will have credence $C r_{p^{\prime}}\left(\bar{e}_{B} / I_{p^{\prime}}\right)=\frac{1}{2}$ no matter what he takes to happen in the backward light cone of $p^{\prime}$ (as specified by $\left.I_{p^{\prime}}\right) .{ }^{25}$ If he accepts quantum theory, Bob will conclude that there is nothing it makes sense to contemplate doing to alter his estimate of $C h_{p^{\prime}}\left(\bar{e}_{B}\right)$, and so there is no conceivable counterfactual scenario in which one in Bob's position arranges for the measurement in $\mathbf{2}$ to have had the different outcome $\bar{e}_{B}$. In general, there is causal dependence between events in $\mathbf{1}$ and $\mathbf{2}$ only if it makes sense to speak of an intervention in one of these regions that would affect a hypothetical agent's estimated chance of what happens in the other. Anyone who accepts quantum theory should deny that makes sense.

By applying quantum theory we can show not only that Bell's particular correlations were to be expected, but also what they depend on. They depend counterfactually but not causally on the quantum state $\Phi^{+}$, and they also depend counterfactually on that state's backing conditions, as described by true magnitude claims. The status of the quantum state disqualifies it from participation in causal relations, but true magnitude claims may be taken to describe beables recognized by quantum theory. To decide which conditions backing any of the states involved in their explanation describe causes of Bell's

\footnotetext{
${ }^{24}$ Since he cannot predict the actual value of $C h_{q}\left(e_{A}\right)$ following his hypothetical polarization measurement, he would have to decide on his best estimate of $C h_{q}\left(e_{A}\right)$ in accordance with Ismael's [38] Ignorance Principle:

Where you're not sure about the chances, form a mixture of the chances assigned by different theories of chance with weights determined by your relative confidence in those theories.

${ }^{25}$ A unitary evolution $\Phi^{+} \Rightarrow \Xi^{+}$corresponding to a local interaction there would still yield $\operatorname{Pr}_{\Xi}+\left(H_{B}\right)=\frac{1}{2}$.
} 
particular correlations or the events they correlate we need further to consider the connection between causation and chance.

The intuition that, other things being equal, a cause raises (or at least alters) the chance of its effect is best cashed out in terms of an interventionist counterfactual: roughly, $c$ is a cause of $e$ just in case $c, e$ are distinct actual events and there is some conceivable intervention on $c$ whose occurrence would have altered the chance of $e$. Such an intervention need not be the act of an agent: it could involve any modification in $c$ of the right kind. Woodward ([63], p. 98) is one influential attempt to say what kind of external influence this would involve. ${ }^{26}$

I used the idea of intervention to argue against any causal dependence between events in $\mathbf{1}$ and $\mathbf{2}$ : anyone who accepts quantum theory accepts that it makes no sense to speak of an intervention on one of these regions that would affect a hypothetical agent's estimated chance of what happens in the other. So even though the outcome $e_{B}$ in $\mathbf{2}$ backs the assignment of state $\left|V_{B}\right\rangle$ to Alice's photon at $q$, the outcome in $\mathbf{1}$ does not depend causally on $e_{B}$ : for similar reasons, neither does the outcome in $\mathbf{2}$ causally depend on that in $\mathbf{1}$. The same idea can now be used to show that both these outcomes do depend causally on whatever event $o$ in the overlap of the backward light cones of $\mathbf{1}$ and 2 warranted assignment of state $\Phi^{+}$—an event truly described by magnitude claims that backed this quantum state.

To keep things simple, assume that the events at which the measurement axes are set on a particular occasion occur in the overlap of the backward light cones of $\mathbf{1}$ and $\mathbf{2}$. Let $r$ be a point outside the future light cones of $e_{A}, e_{B}$ but within the future light cone of the event $o$. Let $e_{A} \uplus e_{B}$ be the event of the joint occurrence of $e_{A}, e_{B}$. This is an event of a type to which the Born rule is applicable: the application yields its chance $C h_{r}\left(e_{A} \uplus e_{B}\right)=P r_{\Phi^{+}}\left(V_{A}, V_{B}\right)=\frac{1}{2}$. Moreover $C h_{r}\left(e_{A}\right)=\operatorname{Pr}_{\Phi^{+}}\left(V_{A}\right)=\frac{1}{2}=\operatorname{Pr}_{\Phi^{+}}\left(V_{B}\right)=C h_{r}\left(e_{B}\right)$. The event $o$ affects all these chances: had a different event $o^{\prime}$ occurred backing the assignment of a different state (e.g. $\left|H_{A}\right\rangle\left|V_{B}\right\rangle$ ), or no event backing any state assignment, then any or all of these chances could have been different. Since it makes sense to speak of an agent altering the chance of event $o$ at $s$ in its past light cone, we have

$$
\begin{aligned}
C h_{r}\left(e_{A} \uplus e_{B} \mid d o-o\right) & \neq C h_{r}\left(e_{A} \uplus e_{B}\right) \\
C h_{r}\left(e_{A} \mid d o-o\right) & \neq C h_{r}\left(e_{A}\right) \\
C h_{r}\left(e_{B} \mid d o-o\right) & \neq C h_{r}\left(e_{B}\right)
\end{aligned}
$$

where $d o-o$ means $o$ is the result of an intervention without which $o$ would not have occurred. It follows that $e_{A}, e_{B}, e_{A} \uplus e_{B}$ are each causally dependent on $o$ : $o$ is a common cause of $e_{A}, e_{B}$ even though the probabilities of events of these

\footnotetext{
${ }^{26}$ Einstein's formulation of a principle of local action also appeals to intervention:
}

The following idea characterizes the relative independence of objects far apart in space ( $A$ and $B$ ): external influence on $A$ has no immediate ("unmittelbar") influence on $B$; this is known as the 'principle of local action' ([14], pp. 321-2) 
types do not factorize. Similar reasoning applies to each registered photon pair on any occasion for any polarization measurement axes. So the second requirement on explanation is met: the separate recording events, as well as the event of their joint occurrence, depend causally on the event $o$ that serves to back assignment of state $\Phi^{+}$to the photon pairs involved in this scenario.

By rejecting any possibility of an intervention expressed by $d o-e_{B}$ or $d o-\bar{e}_{B}$, anyone accepting quantum theory should deny that $C h_{p(q)}\left(e_{A} \mid d o-\right.$ $\left.e_{B}\right) \neq C h_{p(q)}\left(e_{A} \mid d o-\bar{e}_{B}\right)$ is true or even meaningful. Nevertheless $C h_{q}\left(e_{A} \mid e_{B}\right) \neq$ $C h_{q}\left(e_{A} \mid \bar{e}_{B}\right)$ : in this sense $e_{A}$ depends counterfactually but not causally on $e_{B}$.

\section{Conclusion}

Whether quantum entanglement has metaphysical implications sensitively depends on the nature of the quantum state. Schrödinger, who introduced the term, took the implications to be epistemological rather than metaphysical. Einstein used the denial of metaphysical holism and nonseparability in arguing against a view of the quantum state with these implications: But his suggested alternative proved untenable. If viewed as completely describing reality at a fundamental level, entanglement of the quantum state of particles would entail metaphysical nonseparability with respect to ordinary 3-dimensional space, though not with respect to a configuration space of enormous dimension provided this could be taken to be the fundamental space of the world. But entangled quantum states of physically real spacetime regions would imply metaphysical nonseparability in an Everettian view, though this would not be readily apparent within each branch of the multiverse.

Many have followed Schrödinger's characterization of a quantum state as representing not the world but our knowledge of it - complete in the sense that there is nothing more to be known that would enable better predictions than those supplied by Born probabilities. When improved in the light of legitimate objections this leads naturally to a view of quantum states as offering objective prescriptions to (actual or merely hypothetical) physically situated agents, so quantum states are relational but not subjective, and real but not beables. In section 7 I sketched a view of this kind: elsewhere I have called it pragmatist. ${ }^{27}$

A pragmatist seeks to resolve philosophical puzzles that arise when we talk or think about something whose nature is unclear not by asking what in the world it represents but by asking for the function of the associated concept-for what purposes would folk like us have developed a concept like that in a world like this? There is no presumption that the answer to that question is "To represent reality", even if the concept plays a central role in the formulation or application of a fundamental physical theory.

The view of the quantum state sketched in section 7 provides a first illustration of this pragmatist strategy. Viewing quantum states as offering prescriptions and not describing the physical world or our knowledge of it opens

${ }^{27}$ For further details see my ([30], [31], [32], [33]). 
the way to resolution of long-standing conceptual problems of quantum theory, including the measurement problem as well as the problem of non-locality discussed here. Viewed this way a quantum state is real-its prescriptions are objectively (not just intersubjectively) true or false - but it is not an element of physical (or mental) reality.

One can say that a quantum state assignment depends for its truth on backing conditions whose function is to describe the physical world. A pragmatist regards questions of representation as of minor importance. ${ }^{28}$ But if pressed to say what a true quantum state assignment represents it would be better to say it represents the probabilistic relations between its backing conditions and possible states of the world described by the significant magnitude claims which figure in the Born probabilities that result from its application.

While a metaphysician looks to quantum theory to learn what it says chance is at a fundamental level, a pragmatist asks instead how the concept of chance functions in quantum theory as a way of learning why we should have deployed a concept with those features in that context. While a metaphysician asks whether quantum theory locates objective chance as a feature of physical reality at the deepest level, a pragmatist understands the objectivity of chance in quantum theory to derive from its status as a norm to be followed by rational agents whose physical situation renders them informationally deprived.

From this perspective the use of chance in quantum theory makes no radical break with other uses of probability in science as well as daily life. The role of probability was always to present an informationally limited agent with a concise recommendation as to how to set credences concerning matters about which (s)he was not in a position to be certain. What is distinctive about quantum chance is that this recommendation issues directly from application of a fundamental physical theory in situations where fundamental physical theory itself places strict limits on accessible information.

Amateur metaphysicians sometimes express the idea that quantum theory has brought about the downfall of causality, by which they may mean refutation of the view that everything that happens does so by causal necessitythe chain of cause-and-effect works to produce outcomes that are inevitable. The professional metaphysician David Lewis was contemptuous of this idea and (along with many other philosophers) offered an account of probabilistic causation.

Those who know of the strong scientific case for saying that our world is an indeterministic one, and that most events therein are to some extent matters of chance, never seriously renounce the commonsensical view that there is plenty of causation in the world. ([46], p. 217)

On some interpretations (Bohman, Everettian), quantum theory is deterministic: on others (Einstein's "orthodox" view, physical "collapse" theories)

\footnotetext{
${ }^{28}$ As does Huw Price [53] who advocates subject naturalism as an alternative rather than a contribution to metaphysics as currently practised.
} 
it is indeterministic. When the quantum state is understood along the lines of section 7 neither characterization seems appropriate. When relativized to spacetime point $p$ the chance of an event at a time (in some frame) may be 1

while it may be $\frac{1}{2}$ relativized to another spacetime point $q$. It is inappropriate to call such an event inevitable and equally inappropriate to say it is a matter of chance. Better to say it is certain relative to $p$ while maximally uncertain with respect to $q$. Acceptance of this view undercuts the idea that history is produced as a result of the operation of causes, either deterministic or probabilistic.

For a pragmatist, causation is best understood along very similar lines, and not as a physical relation whose nature is to be sought by examining our most fundamental theories. The main reason we have our concept of causal dependence is that we require advice on how to set our credence concerning the possible outcomes of actions we might be contemplating. So to view a relation between events $a, b$ of types $A, B$ from a causal perspective is to evaluate the probability that an event of type $B$ would accompany an event of type $A$ in the hypothetical circumstance that an agent brings about the latter event. One can adopt this perspective while maintaining inability to occupy it or bring about such an event, and even accepting that it would be physically impossible for an agent of any kind to bring about an event of type $A$ in those circumstances. It is this generalized agential perspective that lies behind the application of the notion of an intervention to isolate the kind of counterfactual dependence relation between events that warrants a claim of causal dependence.

By adopting this pragmatist approach to causal dependence one can come to see both why the use of quantum theory to explain Bell's particular correlations involves no instantaneous action at a distance and why it is appropriate to say that events truly described by backing conditions of an entangled quantum state cause the spacelike separated events they explain. In this way one can come to understand those events as effects of a common cause even though their probabilities do not factorize.

A pragmatist can also say why we find this story satisfying as an explanation while still leaving us frustrated about its incompleteness. It has often been remarked that providing a scientific explanation of a puzzling phenomenon can be a uniquely pleasurable experience. But we don't give scientific explanations just for fun: scientific explanations serve two important functions, one epistemic the other practical. By fitting a phenomenon into a wider pattern of similar phenomena that can all be subsumed under theories by modeling them all in very similar ways one can achieve a dramatic economy of thought, just as locating cities and countries on a map enormously helps one to conceptualize their spatial relations. For cognitively limited creatures like us this is an important epistemic function of unifying explanations in science.

Explanations also have a practical function which can be brought out by focusing on their causal aspect. We owe our concept of causation to our limited epistemic and practical abilities. We can find out much about our past by memory, observation and instrumental detection. We can then use scientific theories to attain some warranted beliefs about the future, and with the ability to control through our own actions we can attain more. But, lacking perfect 
control, to exercise this ability effectively we need just the kind of information that is available from the causal perspective - information about the comparative likelihoods of future eventualities conditional on present decisions between alternative actions. That is why we find it overwhelmingly natural to view the world from a causal perspective and why we seek causal explanations whether or not they turn out to amplify our ability to control our future.

Section 8's explanation of puzzling correlations successfully predicted by assignment of an entangled quantum state serves both these epistemic and practical functions. But, because it does not mention any continuous process mediating between that state's backing conditions and the correlated explananda events, it apparently restricts the application of the causal perspective to the spacetime regions to which these pertain, leaving us with nothing to say about intermediate regions. The restriction is not complete: we can model a situation in which something is inserted in the space between source and detector (such as a half-wave plate that is said to rotate the plane of a photon's polarization). But the explanation does not scratch the itch that results from the inability to describe what is happening there, with or without an intervening object or field. Viewing a quantum state as a physical object or property may appear to relieve this itch, but the adverse consequences of such a view show the relief is at best temporary.

\section{References}

[1] Beisbart, C. and Hartmann, S. Probabilities in Physics. Oxford: Oxford University Press (2011).

[2] Bell, J.S. On the Einstein-Podolsky-Rosen paradox. Physics 1 (1964), 447452.

[3] Bell, J.S. On the problem of hidden variables in quantum mechanics. Reviews of Modern Physics 38 (1966), 447-52.

[4] Bell, J.S. Speakable and Unspeakable in Quantum Mechanics, Revised edition. Cambridge: Cambridge University Press (2004).

[5] Bohr, N. The quantum postulate and the recent development of atomic theory. Nature 121 (1927), 580-90.

[6] Bohr, N. Can quantum-mechanical description of physical reality be considered complete? Physical Review 48 (1935), 696-702.

[7] Bohr, N. Unity of knowledge. Reprinted in The Philosophical Writings of Niels Bohr Volume II. Woodbridge, Connecticut: Ox Bow Press (1958).

[8] Caulton, A. Physical entanglement in permutation-invariant quantum mechanics. Available at arXiv: 1409.0246v1 [quant-ph] 31 Aug 2014. 
[9] Clauser, J.F., Holt, R.A., Horne, M.A. and Shimony, A. Proposed experiment to test local hidden-variable theories. Physical Review Letters 23 (1969), 880-4.

[10] Dickson, M. and Clifton, R. Lorentz-invariance in modal interpretations. In The Modal Interpretation of Quantum Mechanics, D. Dieks and P. Vermaas (eds.), Dordrecht: Kluwer Academic Publishers (1998), 9-48.

[11] Dirac, P.A.M. The Principles of Quantum Mechanics. Oxford: Oxford University Press (1930).

[12] Earman, J. Some puzzles and unresolved issues about quantum entanglement. Erkenntnis 80 (2015), 303-337.

[13] Eberhard, P. Bell's theorem and the different conceptions of locality. Il Nuovo Cimento B46 (1978), 392-419.

[14] Einstein, A. Quanten-mechanik und Wirklichkeit. Dialectica 2 (1948), 3204.

[15] Einstein, A. Autobiographical notes; Reply to Criticisms, in P.A. Schilpp (ed.) Albert Einstein: Philosopher-Scientist La Salle, Illinois: Open Court (1949).

[16] Einstein, A., Podolsky, B., and Rosen, N. Can quantum-mechanical description of physical reality be considered complete? Physical Review $\mathbf{4 7}$ (1935), 777-80.

[17] Fine, A. Joint distributions, quantum correlations, and commuting observables. Journal of Mathematical Physics 23 (1982), 1306-10.

[18] Fine, A. The Shaky Game. Chicago: University of Chicago Press (1986).

[19] Fine, A. The Einstein-Podolsky-Rosen argument. The Stanford Encyclopedia of Philosophy (Winter 2014 Edition), Edward N. Zalta (ed.), URL = $<$ http://plato.stanford.edu/archives/win2014/entries/qt-epr/>.

[20] Friederich, S. Rethinking local causality. Synthese 192 (2015), 221-240.

[21] Ghirardi, G.-C. The parts and the whole. Studies in History and Philosophy of Modern Physics 44 (2013), 40-47.

[22] Ghirardi, G.-C., Marinatto, L., and Weber, T. Entanglement and properties of composite systems. Journal of Statistical Physics 108 (2002), 49122 .

[23] Giustina, M. et al. Significant-Loophole-Free Test of Bell's Theorem with Entangled Photons. Physical Review Letters 115, 250401.

[24] Gisin, N. Bell's inequality holds for all non-product states. Physics Letters A154 (1991), 201-2. 
[25] Gleason, A.M. Measures on the closed subspaces of a Hilbert space. Journal of Mathematics and Mechanics 6 (1957), 885-893.

[26] Healey, R. The Philosophy of Quantum Mechanics. Cambridge: Cambridge University Press (1989).

[27] Healey, R. Holism and nonseparability. Journal of Philosophy LXXXVIII (1991), 393-421.

[28] Healey, R. Nonseparable processes and causal explanation", Studies in History and Philosophy of Science 25 (1994), 337-374.

[29] Healey, R. Holism and nonseparabiity in physics. The Stanford Encyclopedia of Philosophy (Spring 2009 Edition), Edward N. Zalta (ed.), URL = $<$ http://plato.stanford.edu/archives/spr2009/entries/physics-holism/>.

[30] Healey, R. Quantum theory: A pragmatist approach. British Journal for the Philosophy of Science 63 (2012), 729-71.

[31] Healey, R. Quantum decoherence in a pragmatist view: Dispelling Feynman's mystery. Foundations of Physics 42 (2012), 1534-55.

[32] Healey, R. Observation and quantum objectivity. Philosophy of Science 80 (2013), 434-53.

[33] Healey, R. How quantum theory helps us explain. British Journal for the Philosophy of Science 66 (2015), 1-43.

[34] Healey, R. Local causality, probability and explanation. In Bell, M. and Gao, S. (eds.) Quantum Nonlocality and Reality. Cambridge: Cambridge University Press (forthcoming).

[35] Henson, J. Non-separability does not relieve the problem of Bell's theorem. Foundations of Physics 43 (2013), 1008-38.

[36] Howard, D. Einstein on locality and separability. Studies in History and Philosophy of Science 16 (1985), 171-201.

[37] Howard, D. Holism, separability and the metaphysical implications of the Bell experiments. in J. Cushing and E. McMullin (eds.) Philosophical Consequences of Quantum Theory: Reflections on Bell's Theorem. Notre Dame, Indiana: University of Notre Dame Press) (1989), 224-53.

[38] Ismael, J. Raid! Dissolving the big, bad bug. Nous 4 (2008), 292-307.

[39] Ismael, J. and Schaffer, J. Quantum holism: Nonseparability as common ground. Synthese (this issue).

[40] Juffman, T. et al.: Wave and particle in molecular interference lithography. Physical Review Letters 103 (2009), 263601. 
[41] Kochen, S. and Specker, E.P. The problem of hidden variables in quantum mechanics. Journal of Mathematics and Mechanics 17 (1967), 59-87.

[42] Ladyman, J., Linnebo, Ø., and Bigaj, T. Entanglement and nonfactorizability. Studies in History and Philosophy of Modern Physics 44 (2013), 215-21.

[43] Lévy-Leblond, J.-M., In K.V. Laurikainen and J. Viiri (eds.) Discussion Sections of the Symposium on the Foundations of Modern Physics 1990. University of Turku.

[44] Leifer, M. Is the quantum state real?", Quanta, 3 (2014), 67-155.

[45] Lewis, D.K. A subjectivist's guide to objective chance. In R.C. Jeffrey (ed.) Studies in Inductive Logic and Probability, Volume II. University of California Press (1980).

[46] Lewis, D.K. Causal explanation. In Philosophical Papers, Volume II. Oxford: Oxford University Press (1986), 214-240.

[47] Maudlin, T. Quantum Non-Locality and Relativity. Third edition. Chichester, West Sussex: Wiley-Blackwell (2011).

[48] Myrvold, W. Modal interpretations and relativity. Foundations of Physics 32 (2001), 1773-84.

[49] Myrvold, W. Relativistic Quantum Becoming. British Journal for the Philosophy of Science $\mathbf{5 3}$ (2003), 475-500.

[50] Mermin, N.D. Quantum Computer Science. Cambridge: Cambridge University Press (2007).

[51] Peirce, C.S. How to Make our Ideas Clear. The Popular Science Monthly January (1878), 286-302.

[52] Price, H. Time's Arrow and Archimedes' Point. Oxford: Oxford University Press (1996).

[53] Price, H. Naturalism Without Mirrors. Oxford: Oxford University Press (2011).

[54] Price, H. Causation, chance and the rational significance of supernatural evidence. Philosophical Review 121 (2012), 483-538.

[55] Schrödinger, E. Discussion of probability relations between separated systems. Mathematical Proceedings of the Cambridge Philosophical Society $\mathbf{3 1}$ (1935), 555-63.

[56] Schrödinger, E. Die gegenwärtige Situation in der Quantenmechanik. Die Naturwissenschaften (1935) 48, 807-812; 49, 823-8; 50, 844-9. 
[57] Schrödinger, E. Probability relations between separated systems. Mathematical Proceedings of the Cambridge Philosophical Society 32 (1936), $446-52$.

[58] Seevinck, M. Monogamy of correlations vs. monogamy of entanglement. Quantum Information Processing 9 (2010), 273-94.

[59] Von Neumann, J. Mathematische Grundlagen der Quantenmechanik. Berlin: Springer (1932).

[60] Wallace, D. and Timpson, C. Quantum mechanics on spacetime I: Spacetime state realism. British Journal for the Philosophy of Science 61 (2010), 697-727.

[61] Wheeler, J.A. and Zurek, W.H. (eds.) Quantum Theory and Measurement. Princeton: Princeton University Press (1983).

[62] Wigner, E. The problem of measurement. American Journal of Physics 31 (1963), 6-15.

[63] Woodward, J. Making Things Happen. Oxford: Oxford University Press (2003). 